\title{
Evaluating the transcriptional fidelity of cancer models
}

Da Peng ${ }^{1 *}$, Rachel Gleyzer ${ }^{2 *}$, Wen-Hsin Tai ${ }^{2}$, Pavithra Kumar $^{2}$, Qin Bian², Bradley Issacs ${ }^{2}$, Edroaldo Lummertz da Rocha ${ }^{3}$, Stephanie Cai ${ }^{1}$, Kathleen DiNapoli ${ }^{4,5}$, Franklin W Huang ${ }^{6}$, Patrick Cahan ${ }^{1,2,7}$

${ }^{1}$ Department of Biomedical Engineering, Johns Hopkins University School of Medicine, Baltimore MD 21205 USA

${ }^{2}$ Institute for Cell Engineering, Johns Hopkins University School of Medicine, Baltimore MD 21205 USA

${ }^{3}$ Department of Microbiology, Immunology and Parasitology, Federal University of Santa Catarina, Florianópolis SC, Brazil

${ }^{4}$ Department of Cell Biology, Johns Hopkins University School of Medicine, Baltimore, MD 21205 USA

${ }^{5}$ Department of Electrical and Computer Engineering, Johns Hopkins University, Baltimore MD 21218 USA

${ }^{6}$ Division of Hematology/Oncology, Department of Medicine; Helen Diller Family Cancer Center; Bakar Computational Health Sciences Institute; Institute for Human Genetics; University of California, San Francisco, San Francisco, CA

${ }^{7}$ Department of Molecular Biology and Genetics, Johns Hopkins University School of Medicine, Baltimore MD 21205 USA

* These authors made equal contributions.

Correspondence to: patrick.cahan@jihmi.edu

Article type: Research

Website: http://www.cahanlab.org/resources/cancerCellNet web

Code: https://github.com/pcahan1/cancerCellNet 


3

\section{ABSTRACT}

Background: Cancer researchers use cell lines, patient derived xenografts, engineered mice, and tumoroids as models to investigate tumor biology and to identify therapies. The generalizability and power of a model derives from the fidelity with which it represents the tumor type under investigation, however, the extent to which this is true is often unclear. The preponderance of models and the ability to readily generate new ones has created a demand for tools that can measure the extent and ways in which cancer models resemble or diverge from native tumors.

Methods: We developed a machine learning based computational tool, CancerCellNet, that measures the similarity of cancer models to 22 naturally occurring tumor types and 36 subtypes, in a platform and species agnostic manner. We applied this tool to 657 cancer cell lines, 415 patient derived xenografts, 26 distinct genetically engineered mouse models, and 131 tumoroids. We validated CancerCellNet by application to independent data, and we tested several predictions with immunofluorescence.

Results: We have documented the cancer models with the greatest transcriptional fidelity to natural tumors, we have identified cancers underserved by adequate models, and we have found models with annotations that do not match their classification. By comparing models across modalities, we report that, on average, genetically engineered mice and tumoroids have higher transcriptional fidelity than patient derived xenografts and cell lines in four out of five tumor types. However, several patient derived xenografts and tumoroids have classification scores that are on par with native tumors, highlighting both their potential as faithful model classes and their heterogeneity.

Conclusions: CancerCellNet enables the rapid assessment of transcriptional fidelity of tumor models. We have made CancerCellNet available as freely downloadable software and as a web application that can be applied to new cancer models that allows for direct comparison to the cancer models evaluated here.

4




\section{INTRODUCTION}

87 Models are widely used to investigate cancer biology and to identify potential therapeutics. Popular modeling modalities are cancer cell lines $(\mathrm{CCLs})^{1}$, genetically engineered mouse models $(\text { GEMMs })^{2}$, patient derived xenografts $(\mathrm{PDXs})^{3}$, and tumoroids ${ }^{4}$. These classes of models differ in the types of questions that they are designed to address. CCLs are often used to address cell intrinsic mechanistic questions ${ }^{5}$, GEMMs to chart progression of molecularly defined-disease ${ }^{6}$, and PDXs to explore patient-specific response to therapy in a physiologically relevant context ${ }^{7}$. More recently, tumoroids have emerged as relatively inexpensive, physiological, in vitro 3D models of tumor epithelium with applications ranging from measuring drug responsiveness to exploring tumor dependence on cancer stem cells. Models also differ in the extent to which the they represent specific aspects of a cancer type ${ }^{8}$. Even with this intraand inter-class model variation, all models should represent the tumor type or subtype under investigation, and not another type of tumor, and not a non-cancerous tissue. Therefore, cancermodels should be selected not only based on the specific biological question but also based on the similarity of the model to the cancer type under investigation ${ }^{9,10}$.

Various methods have been proposed to determine the similarity of cancer models to their intended subjects. Domcke et al devised a 'suitability score' as a metric of the molecular similarity of CCLs to high grade serous ovarian carcinoma based on a heuristic weighting of copy number alterations, mutation status of several genes that distinguish ovarian cancer subtypes, and hypermutation status ${ }^{11}$. Other studies have taken analogous approaches by either focusing on transcriptomic or ensemble molecular profiles (e.g. transcriptomic and copy number alterations) to quantify the similarity of cell lines to tumors ${ }^{12-14}$. These studies were tumor-type specific, focusing on CCLs that model, for example, hepatocellular carcinoma or breast cancer. Notably, Yu et al compared the transcriptomes of CCLs to The Cancer Genome Atlas (TCGA) by correlation analysis, resulting in a panel of CCLs recommended as most 
112

have developed methods to assess CCLs using molecular traits such as copy number alterations (CNA), somatic mutations, DNA methylation and transcriptomics. While all of these studies have provided valuable information, they leave two major challenges unmet. The first challenge is to determine the fidelity of GEMMs, PDXs, and tumoroids, and whether there are stark differences between these classes of models and CCLs. The other major unmet challenge is to enable the rapid assessment of new, emerging cancer models. This challenge is especially relevant now as technical barriers to generating models have been substantially lowered ${ }^{18,19}$, and because new models such as PDXs and tumoroids can be derived on patient-specific basis therefore should be considered a distinct entity requiring individual validation ${ }^{4,20}$.

To address these challenges, we developed CancerCellNet (CCN), a computational tool that uses transcriptomic data to quantitatively assess the similarity between cancer models and 22 naturally occurring tumor types and 36 subtypes in a platform- and species-agnostic manner. Here, we describe CCN's performance, and the results of applying it to assess 657 CCLs, 415 PDXs, 26 GEMMs, and 131 tumoroids. This has allowed us to identify the most faithful models currently available, to document cancers underserved by adequate models, and to find models with inaccurate tumor type annotation. Moreover, because CCN is open-source and easy to use, it can be readily applied to newly generated cancer models as a means to assess their fidelity.

\section{RESULTS}

\section{CancerCellNet classifies samples accurately across species and technologies}

Previously, we had developed a computational tool using the Random Forest classification method to measure the similarity of engineered cell populations to their in vivo counterparts based on transcriptional profiles ${ }^{21,22}$. More recently, we elaborated on this approach to allow for classification of single cell RNA-seq data in a manner that allows for cross-platform and cross-species analysis ${ }^{23}$. Here, we used an analogous approach to build a 
platform that would allow us to quantitatively compare cancer models to naturally occurring patient tumors (Fig 1A). In brief, we used TCGA RNA-seq expression data from 22 solid tumor types to train a top-pair multi-class Random forest classifier (Fig 1B). We combined training data from Rectal Adenocarcinoma (READ) and Colon Adenocarcinoma (COAD) into one COAD_READ category because READ and COAD are considered to be virtually indistinguishable at a molecular level ${ }^{24}$. We included an 'Unknown' category trained using randomly shuffled gene-pair profiles generated from the training data of 22 tumor types to identify query samples that are not reflective of any of the training data. To estimate the performance of $\mathrm{CCN}$ and how it is impacted by parameter variation, we performed a parameter sweep with a 5 -fold $2 / 3$ cross-validation strategy (i.e. $2 / 3$ of the data sampled across each cancer type was used to train, 1/3 was used to validate) (Fig 1C). The performance of CCN, as measured by the mean area under the precision recall curve (AUPRC), did not fall below 0.945 and remained relatively stable across parameter sets (Supp Fig 1A). The optimal parameters resulted in 1,979 features. The mean AUPRCs exceeded 0.95 in most tumor types with this optimal parameter set (Fig 1D, Supp Fig 1B). The AUPRCs of CCN applied to independent data RNA-Seq data from 725 tumors across five tumor types from the International Cancer Genome Consortium (ICGC) ${ }^{25}$ ranged from 0.93 to 0.99 , supporting the notion that the platform is able to accurately classify tumor samples from diverse sources (Fig 1E).

As one of the central aims of our study is to compare distinct cancer models, including GEMMs, our method needed to be able to classify samples from mouse and human samples equivalently. We used the Top-Pair transform ${ }^{23}$ to achieve this and we tested the feasibility of this approach by assessing the performance of a normal (i.e. non-tumor) cell and tissue classifier trained on human data as applied to mouse samples. Consistent with prior applications $^{23}$, we found that the cross-species classifier performed well, achieving mean AUPRC of 0.97 when applied to mouse data (Supp Fig 1C). 
To evaluate cancer models at a finer resolution, we also developed an approach to

164

165

166

167

168

169

170

171

172

173

174

175

176

177

178

179

180

181

182

183

184

185

186

187

188

perform tumor subtype classifications (Supp Fig 1D). We constructed 11 different cancer

subtype classifiers based on the availability of expression or histological subtype

information ${ }^{24,26-36}$. We also included non-cancerous, normal tissues as categories for several subtype classifiers when sufficient data was available: breast invasive carcinoma (BRCA), COAD_READ, head and neck squamous cell carcinoma (HNSC), kidney renal clear cell carcinoma (KIRC) and uterine corpus endometrial carcinoma (UCEC). The 11 subtype classifiers all achieved high overall average AUPRs ranging from 0.80 to 0.99 (Supp Fig 1E).

\section{Fidelity of cancer cell lines}

Having validated the performance of $\mathrm{CCN}$, we then used it to determine the fidelity of CCLs. We mined RNA-seq expression data of 657 different cell lines across 20 cancer types from the Cancer Cell Line Encyclopedia (CCLE) and applied CCN to them, finding a wide classification range for cell lines of each tumor type (Fig 2A, Supp Tab 1). To verify the classification results, we applied CCN to expression profiles from CCLE generated through microarray expression profiling ${ }^{37}$. To ensure that $\mathrm{CCN}$ would function on microarray data, we first tested it by applying a CCN classifier created to test microarray data to 720 expression profiles of 12 tumor types. The cross-platform CCN classifier performed well, based on the comparison to study-provided annotation, achieving a mean AUPRC of 0.91 (Supp Fig 2A). Next, we applied this cross-platform classifier to microarray expression profiles from CCLE (Supp Fig 2B). From the classification results of 571 cell lines that have both RNA-seq and microarray expression profiles, we found a strong overall positive association between the classification scores from RNA-seq and those from microarray (Supp Fig 2C). This comparison supports the notion that the classification scores for each cell line are not artifacts of profiling methodology. Moreover, this comparison shows that the scores are consistent between the times that the cell lines were first assayed by microarray expression profiling in 2012 and by 
RNA-Seq in 2019. We also observed high level of correlation between our analysis and the analysis done by $\mathrm{Yu}$ et $\mathrm{al}^{15}$ (Supp Fig 2D), further validating the robustness of the CCN results. Next, we assessed the extent to which CCN classifications agreed with their nominal tumor type of origin, which entailed translating quantitative CCN scores to classification labels. To achieve this, we selected a decision threshold that maximized the Macro F1 measure, harmonic mean of precision and recall, across 50 cross validations. Then, we annotated cell lines based their $\mathrm{CCN}$ score profile as follows. Cell lines with $\mathrm{CCN}$ scores $>$ threshold for the tumor type of origin were annotated as 'correct'. Cell lines with $\mathrm{CCN}$ scores $>$ threshold in the tumor type of origin and at least one other tumor type were annotated as 'mixed'. Cell lines with $\mathrm{CCN}$ scores $>$ threshold for tumor types other than that of the cell line's origin were annotated as 'other'. Cell lines that did not receive a CCN score > threshold for any tumor type were annotated as 'none' (Fig 2B). We found that majority of cell lines originally annotated as Breast invasive carcinoma (BRCA), Cervical squamous cell carcinoma and endocervical adenocarcinoma (CESC), Skin Cutaneous Melanoma (SKCM), Colorectal Cancer (COAD_READ) and Sarcoma (SARC) fell into the 'correct' category (Fig 2B). On the other hand, no Esophageal carcinoma (ESCA), Pancreatic adenocarcinoma (PAAD) or Brain Lower Grade Glioma (LGG) were classified as 'correct', demonstrating the need for more transcriptionally faithful cell lines that model those general cancer types.

There are several possible explanations for cell lines not receiving a 'correct' classification. One possibility is that the sample was incorrectly labeled in the study from which we harvested the expression data. Consistent with this explanation, we found that colorectal cancer line $\mathrm{NCl}-\mathrm{H} 684^{38,39}$, a cell line labelled as liver hepatocellular carcinoma (LIHC) by CCLE, was classified strongly as COAD_READ (Supp Tab 1). Another possibility to explain low CCN score is that cell lines were derived from subtypes of tumors that are not well-represented in TCGA. To explore this hypothesis, we first performed tumor subtype classification on CCLs from 11 tumor types for which we had trained subtype classifiers (Supp Tab 2). We reasoned that if 
a cell was a good model for a rarer subtype, then it would receive a poor general classification but a high classification for the subtype that it models well. Therefore, we counted the number of

217 lines that fit this pattern. We found that of the 188 lines with no general classification, 25 (13\%)

218 were classified as a specific subtype, suggesting that derivation from rare subtypes is not the 219 major contributor to the poor overall fidelity of CCLs.

Another potential contributor to low scoring cell lines is intra-tumor stromal and immune

221 cell impurity in the training data. If impurity were a confounder of CCN scoring, then we would

expect a strong positive correlation between mean purity and mean CCN classification scores of CCLs per general tumor type. However, the Pearson correlation coefficient between the mean purity of general tumor type and mean CCN classification scores of CCLs in the corresponding general tumor type was low (0.14), suggesting that tumor purity is not a major contributor to the low CCN scores across CCLs (Supp Fig 2E).

\section{Comparison of SKCM and GBM CCLs to sCRNA-seq}

To more directly assess the impact of intra-tumor heterogeneity in the training data on classification approach on scRNA-seq data ${ }^{23}$. Our scRNA-seq classifier achieved a high average AUPRC (0.95) when applied to held-out data and high mean AUPRC (0.99) when applied to few purified bulk testing samples (Supp Fig 3A-B). Comparing the CCN score from bulk RNA-seq general classifier and scRNA-seq classifier, we observed a high level of correlation (Pearson correlation of 0.89 ) between the SKCM CCN classification scores and 237 scRNA-seq SKCM malignant CCN classification scores for SKCM cell lines (Fig 2C, Supp Fig 238 3C). Of the 41 SKCM cell lines that were classified as SKCM by the bulk classifier, 37 were also classified as SKCM malignant cells by the scRNA-seq classifier. Interestingly, we also observed

240 a high correlation between the SARC CCN classification score and scRNA-seq cancer 
associated fibroblast (CAF) CCN classification scores (Pearson correlation of 0.92). Six of the seven SKCM cell lines that had been classified as exclusively SARC by CCN were classified as CAF by the scRNA-seq classifier (Fig 2D, Supp Fig 3C), which suggests the possibility that

244 these cell lines were derived from CAF or other mesenchymal populations, or that they have 245 acquired a mesenchymal character through their derivation. The high level of agreement 246 between scRNA-seq and bulk RNA-seq classification results shows that heterogeneity in the 247 training data of general CCN classifier has little impact in the classification of SKCM cell lines. In contrast, we observed a weaker correlation between GBM CCN classification scores and scRNA-seq GBM neoplastic CCN classification scores (Pearson correlation of 0.72) for GBM cell lines (Fig 2E, Supp Fig 3D). Of the 31 GBM lines that were not classified as GBM with CCN, 25 were classified as GBM neoplastic cells with the scRNA-seq classifier. Among the models suggests that the heterogeneity of glioblastomas ${ }^{42}$ can impact the classification of GBM cell lines, and that the use of scRNA-seq classifier can resolve this deficiency.

\section{Immunofluorescence confirmation of CCN predictions}

To experimentally explore some of our computational analyses, we performed

264 immunofluorescence on three cell lines that were not classified as their labelled categories: the 265 ovarian cancer line SK-OV-3 had a high UCEC CCN score (0.246), the ovarian cancer line 
cancer line PC-3 had a high bladder cancer (BLCA) score (0.307) (Supp Tab 1). We reasoned that if SK-OV-3, A2780 and PC-3 were classified most strongly as UCEC, TGCT and BLCA, respectively, then they would express proteins that are indicative of these cancer types.

First, we measured the expression of the uterine-associated transcription factor $271 \mathrm{HOXB6}^{43,44}$, and the UCEC serous ovarian tumor biomarker WT1 ${ }^{45}$ in SK-OV-3, in the OV cell

272 line Caov-4, and in the UCEC cell line HEC-59. We chose Caov-4 as our positive control for OV 273 biomarker expression because it was determined by our analysis and others ${ }^{11,15}$ to be a good model of OV. Likewise, we chose HEC-59 to be a positive control for UCEC. We found that SKOV-3 has a small percentage $(5 \%)$ of cells that expressed the uterine marker HOXB6 and a large proportion (73\%) of cells that expressed WT1 (Fig 3A). In contrast, no Caov-4 cells expressed HOXB6, whereas $85 \%$ of cells expressed WT1. This suggests that SK-OV-3 exhibits both biomarkers of ovarian tumor and uterine tissue. From our computational analysis and experimental validation, SK-OV-3 is most likely an endometrioid subtype of ovarian cancer. This result is also consistent with prior classification of SK-OV-3 ${ }^{46}$, and the fact that SK-OV-3 lacks p53 mutations, which is prevalent in high-grade serous ovarian cancer ${ }^{47}$, and it harbors an endometrioid-associated mutation in ARID1A ${ }^{11,46,48}$. Next, we measured the expression of markers of OV and germ cell cancers $\left(\right.$ LIN28A $\left.{ }^{49}\right)$ in the OV-annotated cell line A2780, which received a high TCGT CCN score. We found that $54 \%$ of A2780 cells expressed LIN28A whereas it was not detected in Caov-4 (Fig 3B). The OV marker WT1 was also expressed in fewer A2780 cells as compared to Caov-4 (48\% vs $85 \%$ ), which suggests that $A 2780$ could be a germ cell derived ovarian tumor. Taken together, our results suggest that SK-OV-3 and A2780 could represent OV subtypes of that are not well represented in TCGA training data, which resulted in a low OV score and higher $\mathrm{CCN}$ score in other categories.

Lastly, we examined PC-3, annotated as a PRAD cell line but classified to be most

291 similar to BLCA. We found that $30 \%$ of the PC-3 cells expressed PPARG, a contributor to 292 urothelial differentiation ${ }^{50}$ that is not detected in the PRAD Vcap cell line but is highly expressed 
293

in the BLCA RT4 cell line (Fig 3C). PC-3 cells also expressed the PRAD biomarker FOLH1 ${ }^{51}$ suggesting that PC-3 has an PRAD origin and gained urothelial or luminal characteristics through the derivation process. In short, our limited experimental data support the CCN classification results.

\section{Subtype classification of cancer cell lines}

Next, we explored the subtype classification of CCLs from three general tumor types in more depth. We focused our subtype visualization (Fig 4A-C) on CCL models with general CCN score above 0.1 in their nominal cancer type as this allowed us to analyze those models that fell below the general threshold but were classified as a specific sub-type (Supp Tab 1-2). Focusing first on UCEC, the histologically defined subtypes of UCEC, endometrioid and serous, differ in prevalence, molecular properties, prognosis, and treatment. For instance, the endometrioid subtype, which accounts for approximately $80 \%$ of uterine cancers, retains estrogen receptor and progesterone receptor status and is responsive towards progestin therapy ${ }^{52,53}$. Serous, a more aggressive subtype, is characterized by the loss of estrogen and progesterone receptor and is not responsive to progestin therapy ${ }^{52,53}$. CCN classified the majority of the UCEC cell lines as serous except for JHUEM-1 which is classified as mixed, with similarities to both endometrioid and serous (Fig 4A). The preponderance CCLE lines of serous versus endometroid character may be due to properties of serous cancer cells that promote their in vitro propagation, such as upregulation of cell adhesion transcriptional programs ${ }^{54}$. Some of our subtype classification results are consistent with prior observations. For example, HEC-1A, HEC-1B, and KLE were previously characterized as type II endometrial cancer, which includes a serous histological subtype ${ }^{55}$. On the other hand, our subtype classification results contradict prior observations in at least one case. For instance, the Ishikawa cell line was derived from type I endometrial cancer (endometrioid histological subtype) ${ }^{55,56}$, however CCN classified a derivative of this line, Ishikawa 02 ER-, as serous. The high serous CCN score 
could result from a shift in phenotype of the line concomitant with its loss of estrogen receptor

(ER) as this is a distinguishing feature of type II endometrial cancer (serous histological subtype $)^{52}$. Taken together, these results indicate a need for more endometroid-like CCLs.

Next, we examined the subtype classification of Lung Squamous Cell Carcinoma

(LUSC) and Lung adenocarcinoma (LUAD) cell lines (Fig 4B-C). All the LUSC lines with at least one subtype classification had an underlying primitive subtype classification. This is consistent either with the ease of deriving lines from tumors with a primitive character, or with a process by which cell line derivation promotes similarity to more primitive subtype, which is marked by increased cellular proliferation ${ }^{28}$. Some of our results are consistent with prior reports that have investigated the resemblance of some lines to LUSC subtypes. For example, HCC-95, previously been characterized as classical ${ }^{28,57}$, had a maximum CCN score in the classical subtype (0.429). Similarly, LUDLU-1 and EPLC-272H, previously reported as classical ${ }^{57}$ and basal ${ }^{57}$ respectively, had maximal tumor subtype $\mathrm{CCN}$ scores for these sub-types $(0.323$ and proliferation (Fig 4C). RERF-LC-Ad1 had the highest general classification score and the highest proximal inflammation subtype classification score. Taken together, these subtype classification results have revealed an absence of cell lines models for basal and secretory LUSC, and for the Terminal respiratory unit (TRU) LUAD subtype.

\section{Cancer cell lines' popularity and transcriptional fidelity}

Finally, we sought to measure the extent to which cell line transcriptional fidelity related

341 to model prevalence. We used the number of papers in which a model was mentioned,

342 normalized by the number of years since the cell line was documented, as a rough

343 approximation of model prevalence. To explore this relationship, we plotted the normalized

344 citation count versus general classification score, labeling the highest cited and highest 
classified cell lines from each general tumor type (Fig 4D). For most of the general tumor types, the highest cited cell line is not the highest classified cell line except for Hep G2, AGS and ML1, representing liver hepatocellular carcinoma (LIHC), stomach adenocarcinoma (STAD), and thyroid carcinoma (THCA), respectively. On the other hand, the general scores of the highest cited cell lines representing BLCA (T24), BRCA (MDA-MB-231), and PRAD (PC-3) fall below the classification threshold of 0.25 . Notably, each of these tumor types have other lines with scores exceeding 0.5 , which should be considered as more faithful transcriptional models when selecting lines for a study (Supp Tab 1 and

http://www.cahanlab.org/resources/cancerCellNet results/).

\section{Evaluation of patient derived xenografts}

Next, we sought to evaluate a more recent class of cancer models: PDX. To do so, we

subjected the RNA-seq expression profiles of 415 PDX models from 13 different types of cancer types generated previously ${ }^{20}$ to $\mathrm{CCN}$. Similar to the results of CCLs, the PDXs exhibited a wide performed subtype classification on PDX samples: none of the PDX in ESCA were classified as any of the ESCA subtypes (Supp Tab 4). UCEC PDXs had both endometrioid subtypes, serous

367 subtypes, and mixed subtypes, which provided a broader representation than CCLs (Fig 5C).

368 Several LUSC PDXs that were classified as a subtype were also classified as Head and Neck squamous cell carcinoma (HNSC) or mix HNSC and LUSC (Fig 5D). This could be due to the 
consistent with the observation that these PDXs were also subtyped as classical. No LUSC

PDXs were classified as the secretory subtype. In contrast to LUAD CCLs, four of the five LUAD

373 PDXs with a discernible sub-type were classified as proximal inflammatory (Fig 5E). On the

374 other hand, similar to the CCLs, there were no TRU subtypes in the LUAD PDX cohort. In

375 summary, we found that while individual PDXs can reach extremely high transcriptional fidelity

376 to both general tumor types and subtypes, many PDXs were not classified as the general tumor

377 type from which they originated.

\section{Evaluation of GEMMs}

Next, we used CCN to evaluate GEMMs of six general tumor types from nine studies for which expression data was publicly available ${ }^{59-67}$. As was true for CCLs and PDXs, GEMMs also had a wide range of CCN scores (Fig 6A, Supp Tab 5). We next categorized the CCN scores based on the proportion of samples associated with each tumor type that were correctly categorization, GEMMs were invariant. For example, replicates of UCEC GEMMs driven by Prg(cre/+)Pten(lox/lox) received almost identical general CCN scores (Fig 6C, Supp Tab 6).

GEMMs sharing genotypes across studies, such as LUAD GEMMs driven by Kras mutation and loss of p53 ${ }^{59,65,67}$, also received similar general and subtype classification scores (Fig 6 A,B,E).

Next, we explored the extent to which genotype impacted subtype classification in UCEC, LUSC, and LUAD. Prg(cre/+)Pten(lox/lox) GEMMs had a mixed subtype classification of

394 both serous and endometrioid, consistent with the fact that Pten loss occurs in both subtypes

395 (albeit more frequently in endometrioid). We also analyzed Prg(cre/+)Pten(lox/lox)Csf3r-/- 
cancer progression, are depleted in these animals. Interestingly, Prg(cre/+)Pten(lox/lox)Csf3r-/GEMMs had a serous subtype classification, which could be explained by differences in PMN involvement in endometrioid versus serous uterine tumor development that are reflected in the respective transcriptomes of the TCGA UCEC training data. We note that the tumor cells were sorted prior to RNA-seq and thus the shift in subtype classification is not due to contamination of GEMMs with non-tumor components. In short, this analysis supports the argument that tumorcell extrinsic factors, in this case a reduction in anti-tumor PMNs, can shift the transcriptome of

a GEMM so that it more closely resembles a serous rather than endometrioid subtype.

The LUSC GEMMs that we analyzed were Lkb1 ${ }^{\mathrm{fl/fl}}$ and they either overexpressed of Sox2 (via two distinct mechanisms) or were also Pten $^{f / / 1 / 65}$. We note that the eight lenti-Sox2Cre-infected;Lkb1 ${ }^{f l} f^{f l}$ and Rosa26LSL-Sox2-IRES-GFP;Lkb1 $1^{f l} f^{f l}$ samples that classified as 'Unknown' had LUSC CCN scores only modestly lower than the decision threshold (Fig 6D) (mean CCN score $=0.217$ ). Thirteen out of the 17 of the Sox2 GEMMs classified as the secretory subtype of LUSC. The consistency is not surprising given both models overexpress

411 Sox2 and lose Lkb1. On the other hand, the Lkb1/fl/fl:Pten ${ }^{\mathrm{fl} / f l}$ GEMMs had substantially lower general LUSC CCN scores and our subtype classification indicated that this GEMM was mostly classified as 'Unknown', in contrast to prior reports suggesting that it is most similar to a basal subtype $^{68}$. None of the three LUSC GEMMs have strong classical CCN scores. Most of the LUAD GEMMs, which were generated using various combinations of activating Kras mutation, loss of Trp53, and loss of Smarca4L ${ }^{59,65,67}$, were correctly classified (Fig 6E). Those that were 417 not classified have modestly lower CCN score than the decision threshold (mean CCN score $=$ $4180.214)$. There were no substantial differences in general or subtype classification across driver 419 genotypes. Although the sub-type of all LUAD GEMMs was 'Unknown', the subtypes tended to 420 have a mixture of high CCN proximal proliferation, proximal inflammation and TRU scores.

421 Taken together, this analysis suggests that there is a degree of similarity, and perhaps plasticity between the primitive and secretory (but not basal or classical) subtypes of LUSC. On the other 
hand, while the LUAD GEMMs classify strongly as LUAD, they do not have strong particular subtype classification -- a result that does not vary by genotype.

\section{Evaluation of Tumoroids}

Lastly, we used CCN to assess a relatively novel cancer model: tumoroids. We downloaded and assessed 131 distinct tumoroid expression profiles spanning 13 cancer categories from The $\mathrm{NCI}$ Patient-Derived Models Repository (PDMR) ${ }^{69}$ and from three individual studies $^{70-72}$ (Fig 7A, Supp Tab 7). We note that several categories have three or fewer samples (BRCA, CESC, KIRP, OV, LIHC, and BLCA from PDMR). Among the cancer categories represented by more than three samples, only LUSC and PAAD have fewer than $50 \%$ classified as their annotated label (Fig 7B). In contrast to GBM CCLs, all three induced pluripotent stem cell-derived GBM tumoroids ${ }^{72}$ were classified as GBM with high CCN scores (mean $=0.53$ ). To further characterize the tumoroids, we performed subtype classification on them (Supp Tab 8). UCEC tumoroids from PDMR contains a wide range of subtypes with two endometrioid, two serous and one mixed type (Fig 7C). On the other hand, LUSC tumoroids appear to be predominantly of classical subtypes with one tumoroid classified as a mix between classical and primitive (Fig 7D). Lastly, similar to the CCL and PDX counterparts, LUAD tumoroids are classified as proximal inflammatory and proximal proliferation with no tumoroids classified as TRU subtype (Fig 7E).

\section{Comparison of CCLs, PDXs, GEMMs and tumoroids}

Finally, we sought to estimate the comparative transcriptional fidelity of the four cancer models modalities. We compared the general CCN scores of each model on a per tumor type basis (Fig 8). In the case of GEMMs, we used the mean classification score of all samples with shared genotypes. We also used mean classification of technical replicates found in LIHC tumoroids ${ }^{70}$. We evaluated models based on both the maximum CCN score, as this represents 
the potential for a model class, and the median $\mathrm{CCN}$ score, as this indicates the current overall transcriptional fidelity of a model class. PDXs achieved the highest CCN scores in three (UCEC, PAAD, LUAD) out of the five cancer categories in which all four modalities were available (Fig 8), despite having low median CCN scores. Notably, PDXs have a median CCN score above

453 the 0.25 threshold in PAAD while none of the other three modalities have any samples above

454 the threshold. In LIHC, the highest CCN score for PDX (0.9) is only slightly lower than the 455 highest CCN score for tumoroid (0.91). This suggest that certain individual PDXs most closely

456 mimic the transcriptional state of native patient tumors despite a portion of the PDXs having low

457 CCN scores. Similarly, while the majority of the CCLs have low CCN scores, several lines achieve high transcriptional fidelity in LUSC, LUAD and LIHC (Fig 8). Collectively, GEMMs and tumoroids had the highest median CCN scores in four of the five model classes (LUSC and LUAD for GEMMs and UCEC and LIHC for tumoroids). Notably, both of the LIHC tumoroids achieved CCN scores on par with patient tumors (Fig 8). In brief, this analysis indicates that PDXs and CCLs are heterogenous in terms of transcriptional fidelity, with a portion of the models highly mimicking native tumors and the majority of the models having low transcriptional fidelity (with the exception of PAAD for PDXs). On the other hand, GEMMs and tumoroids displayed a consistently high fidelity across different models. gene pairs consisting of 1,689 unique genes) relative to the total number of protein-coding genes in the genome, it is possible that a cancer model with a high $\mathrm{CCN}$ score might not have a 469 high global similarity to a naturally occurring tumor. Therefore, we also calculated the GRN 470 status, a metric of the extent to which tumor-type specific gene regulatory network is 471 established ${ }^{21}$, for all models (Supp Fig 4). We observed high level of correlation between the 472 two similarity metrics, which suggests that although CCN classifies on a selected set of genes, 473 its scores are highly correlated with global assessment of transcriptional similarity. 
We also sought to compare model modalities in terms of the diversity of subtypes that

475 they represent (Supp Fig 5). As a reference, we also included in this analysis the overall

476 subtype incidence, as approximated by incidence in TCGA. Replicates in GEMMs and

477 tumoroids were averaged into one classification profile. In models of UCEC, there is a notable

478 difference in endometroid incidence, and the proportion of models classified as endometroid,

479 with PDX and tumoroids having any representatives (Supp Fig 5). All of the CCL, GEMM, and

480 tumoroid models of PAAD have an unknown subtype classification and no correct general

481 classification. However, the majority of PDXs are subtyped as either a mixture of basal and

482 classical, or classical alone. LUAD have proximal inflammation and proximal proliferation

483 subtypes modelled by CCLs and PDX (Supp Fig 5). Likewise, LUSC have basal, classical and

484 primitive subtypes modelled by CCLs and PDXs, and secretory subtype modelled by GEMMs

exclusively (Supp Fig 5). Taken together, these results demonstrate the need to carefully select

different model systems to more suitably model certain cancer subtypes.

\section{DISCUSSION}

A major goal in the field of cancer biology is to develop models that mimic naturally occurring tumors with enough fidelity to enable therapeutic discoveries. However, methods to measure the extent to which cancer models resemble or diverge from native tumors are lacking. This is especially problematic now because there are many existing models from which to choose, and it has become easier to generate new models. Here, we present CancerCellNet (CCN), a computational tool that measures the similarity of cancer models to 22 naturally occurring tumor

495 types and 36 subtypes. While the similarity of CCLs to patient tumors has already been

496 explored in previous work, our tool introduces the capability to assess the transcriptional fidelity

497 of PDXs, GEMMs, and tumoroids. Because CCN is platform- and species-agnostic, it

498 represents a consistent platform to compare models across modalities including CCLs, PDXs,

499 GEMMs and tumoroids. Here, we applied CCN to 657 cancer cell lines, 415 patient derived 
xenografts, 26 distinct genetically engineered mouse models and 131 tumoroids. Several insights emerged from our computational analyses that have implications for the field of cancer biology.

First, PDXs have the greatest potential to achieve transcriptional fidelity with three out of five general tumor types for which data from all modalities was available, as indicated by the high scores of individual PDXs. Notably PDXs are the only modality with samples classified as PAAD. At the same time, the median CCN scores of PDXs were lower than that of GEMMs and tumoroids in the other four tumor types. It is unclear what causes such a wide range of $\mathrm{CCN}$ scores within PDXs. We suspect that some PDXs might have undergone selective pressures in the host that distort the progression of genomic alterations away from what is observed in natural tumor ${ }^{73}$. Future work to understand this heterogeneity is important so as to yield consistently high fidelity PDXs, and to identify intrinsic and host-specific factors that so powerfully shape the PDX transcriptome.

Second, in general GEMMs and tumoroids have higher median CCN scores than those of PDXs and CCLs. This is also consistent with that fact that GEMMs are typically derived by recapitulating well-defined driver mutations of natural tumors, and thus this observation corroborates the importance of genetics in the etiology of cancer ${ }^{74}$. Moreover, in contrast to most PDXs, GEMMs are typically generated in immune replete hosts. Therefore, the higher overall fidelity of GEMMs may also be a result of the influence of a native immune system on GEMM tumors ${ }^{75}$. The high median CCN scores of tumoroids can be attributed to several factors including the increased mechanical stimuli and cell-cell interactions that come from 3D selforganizing cultures ${ }^{76,77}$.

Third, we have found that none of the samples that we evaluated here are transcriptionally adequate models of ESCA. This may be due to an inherent lability of the ESCA transcriptome that is often preceded by a metaplasia that has obscured determining its cell type(s) of origin ${ }^{78}$. Therefore, this tumor type requires further attention to derive new models. 
Fourth, we found that in several tumor types, GEMMs tend to reflect mixtures of

527 subtypes rather than conforming strongly to single subtypes. The reasons for this are not clear

528 but it is possible that in the cases that we examined the histologically defined subtypes have a

529 degree of plasticity that is exacerbated in the murine host environment.

Lastly, we recognize that many CCLs are not classified as their annotated labels. While

531 we have suggested that the lack of immune component is not a major confounder, we suspect

532 that the CCLs could undergo genetic divergence due to high number of passages,

chemotherapy before biopsy, culture condition and genetic instability ${ }^{79-82}$, which could all be

factors that drive CCLs away from their labelled tumors.

which indicate areas for future work and improvement. First, $\mathrm{CCN}$ is based on transcriptomic

544 incorporate DNA methylation and genomic sequencing data as additional features for our

545 Random forest classifier as this data is becoming more readily available for both training and cancer models. We expect that this will allow us to both refine our tumor subtype categories and

547 it will enable more accurate predictions of how models respond to perturbations such as drug 548 treatment.

A second limitation is that in the cross-species analysis, $\mathrm{CCN}$ implicitly assumes that

550 homologs are functionally equivalent. The extent to which they are not functionally equivalent 
consequence based on the high performance of the normal tissue cross-species classifier and based on the fact that GEMMs have the highest median CCN scores (in addition to tumoroids).

A third caveat to our analysis is that there were many fewer distinct GEMMs and tumoroids than CCLs and PDXs. As more transcriptional profiles for GEMMs and tumoroids emerge, this comparative analysis should be revisited to assess the generality of our results.

Finally, the TCGA training data is made up of RNA-Seq from bulk tumor samples, which necessarily includes non-tumor cells, whereas the CCLs are by definition cell lines of tumor origin. Therefore, CCLs theoretically could have artificially low CCN scores due to the presence of non-tumor cells in the training data. This problem appears to be limited as we found no correlation between tumor purity and CCN score in the CCLE samples. However, this problem is related to the question of intra-tumor heterogeneity. We demonstrated the feasibility of using $\mathrm{CCN}$ and single cell RNA-seq data to refine the evaluation of cancer cell lines contingent upon availability of scRNA-seq training data. As more training single cell RNA-seq data accrues, CCN would be able to not only evaluate models on a per cell type basis, but also based on cellular composition.

We have made the results of our analyses available online so that researchers can easily explore the performance of selected models or identify the best models for any of the 22 general tumor types and the 36 subtypes presented here. To ensure that $\mathrm{CCN}$ is widely available we have developed a free web application, which performs CCN analysis on useruploaded data and allows for direct comparison of their data to the cancer models evaluated here. We have also made the CCN code freely available under an Open Source license and as an easily installed R package, and we are actively supporting its further development. Included in the web application are instructions for training $\mathrm{CCN}$ and reproducing our analysis. The documentation describes how to analyze models and compare the results to the panel of models that we evaluated here, thereby allowing researchers to immediately compare their models to the broader field in a comprehensive and standard fashion. 


\section{Online Methods}

\section{Training General CancerCellNet Classifier}

To generate training data sets, we downloaded 8,991 patient tumor RNA-seq expression count matrix and their corresponding sample table across 22 different tumor types from TCGA using TCGAWorkflowData, TCGAbiolinks ${ }^{85}$ and SummarizedExperiment ${ }^{86}$ packages. We used all the patient tumor samples for training the general CCN classifier. We limited training and analysis of RNA-seq data to the 13,142 genes in common between the TCGA dataset and all the query samples (CCLs, PDXs, GEMMs, and tumoroids). To train the top pair Random forest classifier, we used a method similar to our previous method ${ }^{23} . \mathrm{CCN}$ first normalized the training counts matrix by down-sampling the counts to 500,000 counts per sample. To significantly reduce the execution time and memory of generating gene pairs for all possible genes, CCN then selected $n$ up-regulated genes, $n$ down-regulated genes and $n$ least differentially expressed genes $(\mathrm{CCN}$ training parameter nTopGenes $=n)$ for each of the 22 cancer categories using template matching ${ }^{87}$ as the genes to generate top scoring gene pairs. In short, for each tumor type, CCN defined a template vector that labelled the training tumor samples in cancer type of interest as 1 and all other tumor samples as $0 \mathrm{CCN}$ then calculated the Pearson correlation coefficient between template vector and gene expressions for all genes. The genes with strong match to template as either upregulated or downregulated had large absolute Pearson correlation coefficient. CCN chose the upregulated, downregulated and least differentially expressed genes based on the magnitude of Pearson correlation coefficient.

After CCN selected the genes for each cancer type, CCN generated gene pairs among those genes. Gene pair transformation was a method inspired by the top-scoring pair classifier ${ }^{88}$ to allow compatibility of classifier with query expression profiles that were collected through different platforms (e.g. microarray query data applied to RNA-seq training data). In brief, the gene pair transformation compares 2 genes within an expression sample and encodes the 
604 "gene1_gene2" gene-pair as 1 if the first gene has higher expression than the second gene.

605 Otherwise, gene pair transformation would encode the gene-pair as 0 . Using all the gene pair

606 combinations generated through the gene sets per cancer type, CCN then selected top $m$

607 discriminative gene pairs ( $\mathrm{CCN}$ training parameter nTopGenePairs $=m$ ) for each category using

608 template matching (with large absolute Pearson correlation coefficient) described above. To

609 prevent any single gene from dominating the gene pair list, we allowed each gene to appear at

610 maximum of three times among the gene pairs selected as features per cancer type.

611 After the top discriminative gene pairs were selected for each cancer category, CCN

612 grouped all the gene pairs together and gene pair transformed the training samples into a binary

613 matrix with all the discriminative gene pairs as row names and all the training samples as

614 column names. Using the binary gene pair matrix, CCN randomly shuffled the binary values

615 across rows then across columns to generate random profiles that should not resemble training

616 data from any of the cancer categories. CCN then sampled 70 random profiles, annotated them

617 as "Unknown" and used them as training data for the "Unknown" category. Using gene pair

618 binary training matrix, CCN constructed a multi-class Random Forest classifier of 2000 trees

619 and used stratified sampling of 60 sample size to ensure balance of training data in constructing

620 the decision trees.

To identify the best set of genes and gene-pair parameters ( $n$ and $m$ ), we used a gridsearch cross-validation ${ }^{89}$ strategy with 5 cross-validations at each parameter set. The specific parameters for the final CCN classifier using the function "broadClass_train" in the package cancerCellNet are in Supp Tab 9. The gene-pairs are in Supp Tab 10.

\section{Validating General CancerCellNet Classifier}

Two thirds of patient tumor data from each cancer type were randomly sampled as

628 training data to construct a $\mathrm{CCN}$ classifier. Based on the training data, CCN selected the 629 classification genes and gene-pairs and trained a classifier. After the classifier was built, 35 
held-out samples from each cancer category were sampled and 40 "Unknown" profiles were generated for validation. The process of randomly sampling training set from $2 / 3$ of all patient tumor data, selecting features based on the training set, training classifier and validating was repeated 50 times to have a more comprehensive assessment of the classifier trained with the optimal parameter set. To test the performance of final CCN on independent testing data, we applied it to 725 profiles from ICGC spanning 6 projects that do not overlap with TCGA (BRCAKR, LIRI-JP, OV-AU, PACA-AU, PACA-CA, PRAD-FR).

\section{Selecting Decision Thresholds}

Our strategy for selecting a decision threshold was to find the value that maximizes the average Macro F1 measure ${ }^{90}$ for each of the 50 cross-validations that were performed with the optimal parameter set, testing thresholds between 0 and 1 with a 0.01 increment. The F1 measure is defined as:

$$
\text { Macro } F 1=\frac{2 \times \text { precision } \times \text { recall }}{\text { precision }+ \text { recall }}
$$

644 We selected the most commonly occurring threshold above 0.2 that maximized the average

645 Macro F1 measure across the 50 cross-validations as the decision threshold for the final 646 classifier (threshold $=0.25$ ). The same approach was applied for the subtype classifiers. The

647 thresholds and the corresponding average precision, recall and F1 measures are recorded in 648 (Supp Tab 11).

\section{Classifying Query Data into General Cancer Categories}

We downloaded the RNA-seq cancer cell lines expression profiles and sample table from (https://portals.broadinstitute.org/ccle/data), and microarray cancer cell lines expression 
annotations from the authors of Gao et al ${ }^{20}$. We gathered GEMM expression profiles from nine different studies $^{59-67}$. We downloaded tumoroid expression profiles from The NCI PatientDerived Models Repository (PDMR) $)^{69}$ and from three individual studies ${ }^{70-72}$. To use CCN classifier on GEMM data, the mouse genes from GEMM expression profiles were converted into

659 their human homologs. The query samples were classified using the final CCN classifier. Each 660 query classification profile was labelled as one of the four classification categories: "correct", 661 "mixed", "none" and "other" based on classification profiles. If a sample has a CCN score higher than the decision threshold in the labelled cancer category, we assigned that as "correct". If a sample has $\mathrm{CCN}$ score higher than the decision threshold in labelled cancer category and in other cancer categories, we assigned that as "mixed". If a sample has no CCN score higher than the decision threshold in any cancer category or has the highest CCN score in 'Unknown' category, then we assigned it as "none". If a sample has CCN score higher than the decision pheatmap ${ }^{92}$ and ggplot $2^{93}$.

\section{Cross-Species Assessment}

To assess the performance of cross-species classification, we downloaded 1003

673 labelled human tissue/cell type and 1993 labelled mouse tissue/cell type RNA-seq expression

674 profiles from Github (https://github.com/pcahan1/CellNet). We first converted the mouse genes

675 into human homologous genes. Then we found the intersecting genes between mouse

676 tissue/cell expression profiles and human tissue/cell expression profiles. Limiting the input of

677 human tissue RNA-seq profiles to the intersecting genes, we trained a CCN classifier with all

678 the human tissue/cell expression profiles. The parameters used for the function

679 "broadClass_train" in the package cancerCellNet are in Supp Tab 9. We randomly sampled 75 
samples from each tissue category in mouse tissue/cell data and applied the classifier on those samples to assess performance.

\section{Cross-Technology Assessment}

To assess the performance of CCN in applications to microarray data, we gathered 6,219 patient tumor microarray profiles across 12 different cancer types from more than 100 different projects (Supp Tab 12). We found the intersecting genes between the microarray profiles and TCGA patient RNA-seq profiles. Limiting the input of RNA-seq profiles to the intersecting genes, we created a CCN classifier with all the TCGA patient profiles using parameters for the function "broadClass_train" listed in Supp Tab 9. After the microarray specific classifier was trained, we randomly sampled 60 microarray patient samples from each cancer category and applied CCN classifier on them as assessment of the cross-technology performance in Supp Fig 2A. The same CCN classifier was used to assess microarray CCL samples Supp Fig 2B.

\section{Training and validating scRNA-seq Classifier}

We extracted labelled human melanoma and glioblastoma scRNA-seq expression profiles $^{40,41}$, and compiled the two datasets excluding 3 cell types T.CD4, T.CD8 and Myeloid due to low number of cells for training. 60 cells from each of the 11 cell types were sampled for training a scRNA-seq classifier. The parameters for training a general scRNA-seq classifier using the function "broadClass_train" are in Supp Tab 9. 25 cells from each of the 11 cell types from the held-out data were selected to assess the single cell classifier. Using maximization of average Macro F1 measure, we selected the decision threshold of 0.255 . The gene-pairs that were selected to construct the classifier are in Supp Tab 10. To assess the cross-technology capability of applying scRNA-seq classifier to bulk RNA-seq, we downloaded 305 expression 
705

706

707

708

709

710

711

712

713

714

715

716

717

718

719

720

721

722

723

724

725

726

727

728

729

730

profiles spanning 4 purified cell types (B cells, endothelial cells, monocyte/macrophage, fibroblast) from https://github.com/pcahan1/CellNet.

\section{Training Subtype CancerCellNet}

We found 11 cancer types (BRCA, COAD, ESCA, HNSC, KIRC, LGG, PAAD, UCEC, STAD, LUAD, LUSC) which have meaningful subtypes based on either histology or molecular profile and have sufficient samples to train a subtype classifier with high AUPR. We also included normal tissues samples from BRCA, COAD, HNSC, KIRC, UCEC to create a normal tissue category in the construction of their subtype classifiers. Training samples were either labelled as a cancer subtype for the cancer of interest or as "Unknown" if they belong to other cancer types. Similar to general classifier training, CCN performed gene pair transformation and selected the most discriminate gene pairs for each cancer subtype. In addition to the gene pairs selected to discriminate cancer subtypes, CCN also performed general classification of all training data and appended the classification profiles of training data with gene pair binary matrix as additional features. The reason behind using general classification profile as additional features is that many general cancer types may share similar subtypes, and general classification profile could be important features to discriminate the general cancer type of interest from other cancer types before performing finer subtype classification. The specific parameters used to train individual subtype classifiers using "subClass_train" function of CancerCellNet package can be found in Supp Tab 9 and the gene pairs are in Supp Tab 10.

\section{Validating Subtype CancerCellNet}

Similar to validating general class classifier, we randomly sampled $2 / 3$ of all samples in each cancer subtype as training data and sampled an equal amount across subtypes in the $1 / 3$ held-out data for assessing subtype classifiers. We repeated the process 20 times for more comprehensive assessment of subtype classifiers. 


\section{Classifying Query Data into Subtypes}

We assigned subtype to query sample if the query sample has CCN score higher than the decision threshold. The table of decision threshold for subtype classifiers are in Supp Tab 11. If no CCN scores exceed the decision threshold in any subtype or if the highest CCN score is in 'Unknown' category, then we assigned that sample as 'Unknown'. Analysis was performed in $\mathrm{R}$ and visualizations were generated with the ComplexHeatmap package ${ }^{94}$.

\section{Cells culture, Immunohistochemistry and histomorphometry}


NCCIT(ATCC® CRL-2073 ${ }^{\text {TM }}$ ) cell lines were purchased from ATCC. HEC-59 (C0026001) and A2780 (93112519-1VL) were obtained from Addexbio Technologies and Sigma-Aldrich. Vcap and PC-3. SK-OV-3, Vcap, and RT4 were cultured in Dulbecco's Modified Eagle Medium (DMEM, high glucose, 11960069, Gibco) with 1\% Penicillin-Streptomycin-Glutamine ( 10378016, Life Technologies); Caov-4, PC-3, NCCIT, and A2780 were cultured using RPMI1640 medium (11875093, Gibco) while HEC-59 was in Iscove's Modified Dulbecco's Medium (IMDM, 12440053, Gibco). Both media were supplemented with 1\% Penicillin-Streptomycin (15140122, Gibco). All medium included 10\% Fetal Bovine Serum (FBS).

Cells cultured in 48-well plate were washed twice with PBS and fixed in $10 \%$ buffered formalin for $24 \mathrm{hrs}$ at $4{ }^{\circ} \mathrm{C}$. Immunostaining was performed using a standard protocol. Cells were incubated with primary antibodies to goat HOXB6 (10 $\mu \mathrm{g} / \mathrm{mL}$, PA5-37867, Invitrogen),

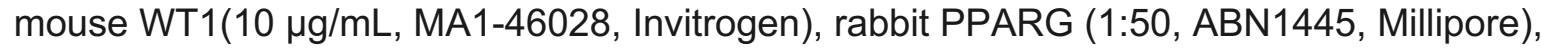
mouse FOLH1(10 $\mu \mathrm{g} / \mathrm{mL}$, UM570025, Origene), and rabbit LIN28A (1:50, \#3978, Cell Signaling) in Antibody Diluent (S080981-2, DAKO), at $4{ }^{\circ} \mathrm{C}$ overnight followed with three 5 min washes in TBST. The slides were then incubated with secondary antibodies conjugated with fluorescence at room temperature for $1 \mathrm{~h}$ while avoiding light followed with three 5 min washes in TBST and 
nuclear stained with mounting medium containing DAPI. Images were captured by Nikon EcLipse Ti-S, DS-U3 and DS-Qi2.

Histomorphometry was performed using ImageJ (Version 2.0.0-rc-69/1.52i). \%

N.positive cells was calculated by the percentage of the number of positive stained cells divided by the number of DAPI-positive nucleus within three of randomly chosen areas. The data were expressed as means \pm SD.

\section{Tumor Purity Analysis}

We used the R package ESTIMATE ${ }^{95}$ to calculate the ESTIMATE scores from TCGA tumor expression profiles that we used as training data for $\mathrm{CCN}$ classifier. To calculate tumor purity we used the equation described in YoshiHara et al., 2013 ${ }^{95}$ :

$$
\text { Tumour purity }=\cos (0.6049872018+0.0001467884 \times \text { ESTIMATE score })
$$

\section{Extracting Citation Counts}

We used the R package RISmed ${ }^{96}$ to extract the number of citations for each cell line through query search of "cell line name[Text Word] AND cancer[Text Word]" on PubMed. The citation counts were normalized by dividing the citation counts with the number of years since first documented.

$$
\text { Normalized citation counts }=\frac{\text { citation counts }}{\# \text { years since first documented }}
$$

\section{GRN construction and GRN Status}

GRN construction was extended from our previous method ${ }^{21} .80$ samples per cancer type were randomly sampled and normalized through down sampling as training data for the CLR GRN construction algorithm. Cancer type specific GRNs were identified by determining the 
differentially expressed genes per each cancer type and extracting the subnetwork using those genes.

To extend the original GRN status algorithm ${ }^{21}$ across different platforms and species, we devised a rank-based GRN status algorithm. Like the original GRN status, rank based GRN status is a metric of assessing the similarity of cancer type specific GRN between training data in the cancer type of interest and query samples. Hence, high GRN status represents high level of establishment or similarity of the cancer specific GRN in the query sample compared to those of the training data. The expression profiles of training data and query data were transformed into rank expression profiles by replacing the expression values with the rank of the expression values within a sample (highest expressed gene would have the highest rank and lowest expressed genes would have a rank of 1). Cancer type specific mean and standard deviation of every gene's rank expression were learned from training data. The modified Z-score values for genes within cancer type specific GRN were calculated for query sample's rank expression profiles to quantify how dissimilar the expression values of genes in query sample's cancer type specific GRN compared to those of the reference training data:

$$
Z \text { score }(\text { gene } i)_{\text {mod }}=\left\{\begin{array}{c}
0, \text { if Zscore is positive and the gene is found to be upregulated } \\
0, \text { if Zscore is negative and the gene is found to be downregulated } \\
\text { abs }(Z \text { score }), \text { otherwise }
\end{array}\right.
$$

If a gene in the cancer type specific GRN is found to be upregulated in the specific cancer type relative to other cancer types, then we would consider query sample's gene to be similar if the ranking of the query sample's gene is equal to or greater than the mean ranking of the gene in training sample. As a result of similarity, we assign that gene of a Z-score of 0 . The same principle applies to cases where the gene is downregulated in cancer specific subnetwork. GRN status for query sample is calculated as the weighted mean of the $\left(1000-Z\right.$ score $\left.(\text { gene } i)_{\text {mod }}\right)$ across genes in cancer type specific GRN. 1000 is an arbitrary 
large number, and larger dissimilarity between query's cancer type specific GRN indicate high

804

805

806

807

808

809

810

\section{Code availability}

818 CancerCellNet code and documentation is available at GitHub:

819 https://github.com/pcahan1/cancerCellNet

$$
\begin{gathered}
R G S=\sum_{i=1}^{n}\left(1000-Z_{\text {score }}(\text { gene } i)_{\text {mod }}\right) \text { weight } t_{\text {gene } i} \\
\text { GRN Status }=\frac{R G S}{\sum_{i=1}^{n} \text { weight }_{\text {gene } i}}
\end{gathered}
$$
mean GRN status.

\section{Acknowledgements \\ Acknowledgements}

822 This work was supported by the National Institutes of Health NCI Ovarian Cancer SPORE 823 P50CA228991 via a Development Research Program award to PC. FWH was supported by a 824 Prostate Cancer Foundation Young Investigator Award, Department of Defense W81XWH-17-

The weight of individual genes in the cancer specific network is determined by the importance of the gene in the Random Forest classifier. Finally, the GRN status gets normalized with respect to the GRN status of the cancer type of interest and the cancer type with the lowest

$$
\text { Normalized GRN status }=\frac{G R N \text { status } \text { query }-\operatorname{avg}(G R N \text { status } \text { min cancer })}{\operatorname{avg}\left(G R N \text { status }_{\text {cancer } \text { type interest }}\right)}
$$

Where "min cancer" represents the cancer type where its training data have the lowest mean GRN status in the cancer type of interest, and avg (GRN status min cancer) represents the lowest average GRN status in the cancer type of interest. avg(GRN status cancer type interest $)$ represents average GRN status of the cancer type of interest in the training data.

820 
01 (F.W.H.) U19 CA214253 (F.W.H.). We would like to thank John Powers, Hao Zhu, Tian-Li

827

828

829

830

831

832

833

834

835

836

837

838

839

840

841

842

843

844

845

846

847

848

849

850

851

Wang, Charles Eberhart, and Kaloyan Tsanov for comments on the manuscript and helpful discussions. Some figures were created in part with Biorender.com.

\section{FIGURE LEGENDS}

Fig. 1 CancerCelINet (CCN) workflow, training, and performance. (A) Schematic of CCN usage. CCN was designed to assess and compare the expression profiles of cancer models such as CCLs, PDXs, GEMMs, and tumoroids with native patient tumors. To use trained classifier, CCN inputs the query samples (e.g. expression profiles from CCLs, PDXs, GEMMs, tumoroids) and generates a classification profile for the query samples. The column names of the classification heatmap represent sample annotation and the row names of the classification heatmap represent different cancer types. Each grid is colored from black to yellow representing the lowest classification score (e.g. 0) to highest classification score (e.g. 1). (B) Schematic of $\mathrm{CCN}$ training process. $\mathrm{CCN}$ uses patient tumor expression profiles of 22 different cancer types from TCGA as training data. First, CCN identifies $n$ genes that are upregulated, $n$ that are downregulated, and $n$ that are relatively invariant in each tumor type versus all of the others.

Then, CCN performs a pair transform on these genes and subsequently selects the most discriminative set of $m$ gene pairs for each cancer type as features (or predictors) for the Random forest classifier. Lastly, CCN trains a multi-class Random Forest classifier using genepair transformed training data. (C) Parameter optimization strategy. 5 cross-validations of each parameter set in which $2 / 3$ of TCGA data was used to train and $1 / 3$ to validate was used search for the values of $n$ and $m$ that maximized performance of the classifier as measured by area under the precision recall curve (AUPRC). (D) Mean and standard deviation of classifiers based on 50 cross-validations with the optimal parameter set. (E) AUPRC of the final CCN classifier when applied to independent patient tumor data from ICGC. 
Fig. 2 Evaluation of cancer cell lines. (A) General classification heatmap of CCLs extracted from CCLE. Column annotations of the heatmap represent the labelled cancer category of the CCLs given by CCLE and the row names of the heatmap represent different cancer categories. CCLs' general classification profiles are categorized into 4 categories: correct (red), correct mixed (pink), no classification (light green) and other classification (dark green) based on the decision threshold of 0.25 . (B) Bar plot represents the proportion of each classification category in CCLs across cancer types ordered from the cancer types with the highest proportion of correct and correct mixed CCLs to lowest proportion. (C) Comparison between SKCM general CCN scores from bulk RNA-seq classifier and SKCM malignant CCN scores from scRNA-seq classifier for SKCM CCLs. (D) Comparison between SARC general CCN scores from bulk RNAseq classifier and CAF CCN scores from scRNA-seq classifier for SKCM CCLs. (E) Comparison between GBM general CCN scores from bulk RNA-seq classifier and GBM neoplastic CCN scores from scRNA-seq classifier for GBM CCLs. (F) Comparison between SARC general CCN scores and CAF CCN scores from scRNA-seq classifier for GBM CCLs. The green lines indicate the decision threshold for scRNA-seq classifier and general classifier.

Fig. 3 Immunofluorescence of selected cell lines. (A) Classification profiles (left) and IF expression (middle) of Caov-4 (OV positive control), HEC-59 (UCEC positive control) and SKOV-3 for WT1 (OV biomarker) and HOXB6 (uterine biomarker). The bar plots quantify the average percentage of positive cells for WT1 (top-right) and HOXB6 (bottom-right). (B)

872 Classification profiles (left) and IF expression (middle) of Caov-4, NCCIT (germ cell tumor 873 positive control) and A2780 for WT1 and LIN28A (germ cell tumor biomarker). Classification of 874 NCCIT were performed using RNA-seq profiles of WT control NCCIT duplicate from Grow et $875 \mathrm{al}^{91}$. The bar plots quantify the average percentage of positive cells for WT1 (top-right) and 876 LIN28A (bottom-right). (C) Classification profiles (left) and IF expression (middle) of Vcap 877 (PRAD positive control), RT4 (BLCA positive control) and PC-3 for FOLH1 (prostate biomarker) 
and PPARG (urothelial biomarker). The bar plots quantify the average percentage of positive cells for FOLH1 (top-right) and PPARG (bottom-right).

Fig. 4 Subtype classification of CCLs and CCL prevalence. The heatmap visualizations represent subtype classification of (A) UCEC CCLs, (B) LUSC CCLs and (C) LUAD CCLs. Only samples with CCN scores $>0.1$ in their nominal tumor type are displayed. (D) Comparison of normalized citation counts and general CCN classification scores of CCLs. Labelled cell lines either have the highest $\mathrm{CCN}$ classification score in their labelled cancer category or highest normalized citation count. Each citation count was normalized by number of years since first documented on PubMed.

Fig. 5 Evaluation of patient derived xenografts. (A) General classification heatmap of PDXs. Column annotations represent annotated cancer type of the PDXs, and row names represent cancer categories. (B) Proportion of classification categories in PDXs across cancer types is visualized in the bar plot and ordered from the cancer type with highest proportion of correct and mixed correct classified PDXs to the lowest. Subtype classification heatmaps of (C) UCEC PDXs, (D) LUSC PDXs and (E) LUAD PDXs. Only samples with CCN scores $>0.1$ in their nominal tumor type are displayed.

\section{Fig. 6 Evaluation of genetically engineered mouse models. (A) General classification} heatmap of GEMMs. Column annotations represent annotated cancer type of the GEMMs, and row names represent cancer categories. (B) Proportion of classification categories in GEMMs across cancer types is visualized in the bar plot and ordered from the cancer type with highest proportion of correct and mixed correct classified GEMMs to the lowest. Subtype classification heatmap of (C) UCEC GEMMs, (D) LUSC GEMMs and (E) LUAD GEMMs. Only samples with CCN scores $>0.1$ in their nominal tumor type are displayed. 
Fig. 7 Evaluation of tumoroid models. (A) General classification heatmap of tumoroids.

Column annotations represent annotated cancer type of the tumoroids, and row names

907 represent cancer categories. (B) Proportion of classification categories in tumoroids across

908 cancer types is visualized in the bar plot and ordered from the cancer type with highest

909 proportion of correct and mixed correct classified tumoroids to the lowest. Subtype classification

910 heatmap of (C) UCEC tumoroids, (D) LUSC tumoroids and (E) LUAD tumoroids. Only samples

911 with CCN scores $>0.1$ in their nominal tumor type are displayed.

913 Fig. 8 Comparison of CCLs, PDXs, and GEMMs. Box-and-whiskers plot comparing general

914 CCN scores across CCLs, GEMMs, PDXs of five general tumor types (UCEC, PAAD, LUSC, 915 LUAD, LIHC).

\section{Supplementary Information}

918 Supplementary Figure 1 Assessment of CCN general classifier and subtype classifier. (A)

919 Mean AUPRC of repeated grid-search cross-validation for each parameter grid. (B) Mean and 920 range of CCN classifier's PR curves from 50 cross validations based on the optimal feature 921 selection parameters $n$ and $m$. (C) AUPRC of CCN human tissue classifier when applied to 922 mouse tissue data. (D) The schematic of training a subtype classifier in CCN. CCN uses patient 923 tumor expression profiles from cancer of interest as training data. CCN performs gene-pair 924 transformation and selects the most discriminative gene pairs among the cancer subtypes from 925 training data as features. CCN then applies the general classification on training data and uses 926 the general classification profile as features in addition to gene pairs for training a Random 927 Forest classifier. The weight of the general classification profiles as features can be tuned to 928 improve AUPRC. (E) The mean and standard deviation of AUPRC for 11 subtype classifiers based on 20 iterations of random sampling of training and held-out data, training subtype 
classifier using training data, classification of held-out data, and calculation of recall and precision.

Supplementary Figure 2 Further validation of $\mathrm{CCN}$ and classification results. To validate the cross-platform classification performance of $\mathrm{CCN}$, a new classifier specifically trained to classify microarray data was trained using RNA-seq data from TCGA as training data and intersecting genes between RNA-seq data and microarray data. (A) AUPRC of CCN classifier when applied to tumor profiles assayed on microarrays. (B) Classification heatmap of CCLs using microarray expression data. (C) Pearson correlation between CCN scores of CCLE lines generated from RNA-seq data and microarray data. (D) Comparison between CCLs' CCN scores and the similarity metric from $\mathrm{Yu}$ et $\mathrm{al}^{15}$, median correlations of transcriptional profiles between CCLs and TCGA tumors from CCLs' labelled cancer category. (E) Comparison of mean tumor purity of training data and mean CCN scores of CCLs for each cancer category.

Supplementary Figure 3 Single-cell classification of SKCM and GBM cell lines. (A) AUPRC of the single-cell classifier when applied to scRNA-seq held-out data. (B) AUPRC of the scRNAseq classifier when applied to purified bulk RNA samples. (C) Single-cell classification of SKCM CCLs. Red bar-plot (top) represents general CCN scores in SARC and blue bar-plot (bottom) represents general CCN scores in SKCM. (D) Single-cell classification of GBM CCLs. Red barplot (top) represents general CCN scores in SARC and yellow bar-plot (bottom) represents general CCN scores in GBM.

Supplementary Figure 4 Correlation between cancer type specific network GRN status and general CCN scores.

Supplementary Figure 5 Proportion of cancer subtypes in different cancer models and TCGA tumor data across 11 general cancer types. 
959

960

961

962

963

964

965

966

967

968

969

970

971

972

973

974

975

976

977

978

979

980

981

982

983

984

985

986

987

988

989

990

991

992

993

994

995

996

997

998

999

1000

1001

1002

1003

1004

Supplementary Table 1 General classification profiles of CCLs.

Supplementary Table 2 Subtype classification profiles of CCLs.

Supplementary Table 3 General classification profiles of PDXs.

Supplementary Table 4 Subtype classification profiles of PDXs.

Supplementary Table 5 General classification profiles of GEMMs

Supplementary Table 6 Subtype classification profiles of GEMMs.

Supplementary Table 7 General classification profiles of tumoroids.

Supplementary Table 8 Subtype classification profiles of tumoroids.

Supplementary Table 9 Specific parameters used for training of all classifiers.

Supplementary Table 10 Gene-pairs selected for final training of CCN general, subtype classifiers and single-cell classifier.

Supplementary Table 11 Decision thresholds and the corresponding precision and recall for the general classifier and subtype classifier.

Supplementary Table 12 Accessions of tumor microarray data used in validation.

\section{REFERENCES}

1. Sharma, S. V., Haber, D. A. \& Settleman, J. Cell line-based platforms to evaluate the therapeutic efficacy of candidate anticancer agents. Nat. Rev. Cancer 10, 241253 (2010).

2. Kersten, K., de Visser, K. E., van Miltenburg, M. H. \& Jonkers, J. Genetically engineered mouse models in oncology research and cancer medicine. EMBO Mol. Med. 9, 137-153 (2017).

3. Hidalgo, M. et al. Patient-derived xenograft models: an emerging platform for translational cancer research. Cancer Discov. 4, 998-1013 (2014).

4. Drost, J. \& Clevers, H. Organoids in cancer research. Nat. Rev. Cancer 18, 407418 (2018).

5. Klijn, C. et al. A comprehensive transcriptional portrait of human cancer cell lines. Nat. Biotechnol. 33, 306-312 (2015).

6. Koren, S. et al. PIK3CA(H1047R) induces multipotency and multi-lineage mammary tumours. Nature 525, 114-118 (2015).

7. DeRose, Y. S. et al. Tumor grafts derived from women with breast cancer authentically reflect tumor pathology, growth, metastasis and disease outcomes. Nat. Med. 17, 1514-1520 (2011). 
1005

1006

1007

1008

1009

1010

1011

1012

1013

1014

1015

1016

1017

1018

1019

1020

1021

1022

1023

1024

1025

1026

1027

1028

1029

1030

1031

1032

1033

1034

1035

1036

1037

1038

1039

1040

1041

1042

1043

1044

1045

1046

1047

1048

1049

1050
8. Sharpless, N. E. \& Depinho, R. A. The mighty mouse: genetically engineered mouse models in cancer drug development. Nat. Rev. Drug Discov. 5, 741-754 (2006).

9. Mouradov, D. et al. Colorectal cancer cell lines are representative models of the main molecular subtypes of primary cancer. Cancer Res. 74, 3238-3247 (2014).

10. Stuckelberger, S. \& Drapkin, R. Precious GEMMs: emergence of faithful models for ovarian cancer research. J. Pathol. 245, 129-131 (2018).

11. Domcke, S., Sinha, R., Levine, D. A., Sander, C. \& Schultz, N. Evaluating cell lines as tumour models by comparison of genomic profiles. Nat. Commun. 4, 2126 (2013).

12. Jiang, G. et al. Comprehensive comparison of molecular portraits between cell lines and tumors in breast cancer. BMC Genomics 17 Suppl 7, 525 (2016).

13. Chen, B., Sirota, M., Fan-Minogue, H., Hadley, D. \& Butte, A. J. Relating hepatocellular carcinoma tumor samples and cell lines using gene expression data in translational research. BMC Med. Genomics 8 Suppl 2, S5 (2015).

14. Vincent, K. M., Findlay, S. D. \& Postovit, L. M. Assessing breast cancer cell lines as tumour models by comparison of mRNA expression profiles. Breast Cancer Res. 17, 114 (2015).

15. $\mathrm{Yu}, \mathrm{K}$. et al. Comprehensive transcriptomic analysis of cell lines as models of primary tumors across 22 tumor types. Nat. Commun. 10, 3574 (2019).

16. Najgebauer, H. et al. CELLector: Genomics-Guided Selection of Cancer In Vitro Models. Cell Syst. 10, 424-432.e6 (2020).

17. Salvadores, M., Fuster-Tormo, F. \& Supek, F. Matching cell lines with cancer type and subtype of origin via mutational, epigenomic, and transcriptomic patterns. Sci. Adv. 6, (2020).

18. Guernet, A. \& Grumolato, L. CRISPR/Cas9 editing of the genome for cancer modeling. Methods 121-122, 130-137 (2017).

19. Gargiulo, G. Next-Generation in vivo Modeling of Human Cancers. Front. Oncol. 8, 429 (2018).

20. Gao, H. et al. High-throughput screening using patient-derived tumor xenografts to predict clinical trial drug response. Nat. Med. 21, 1318-1325 (2015).

21. Cahan, P. et al. CellNet: network biology applied to stem cell engineering. Cell 158, 903-915 (2014).

22. Radley, A. H. et al. Assessment of engineered cells using CellNet and RNA-seq. Nat. Protoc. 12, 1089-1102 (2017).

23. Tan, Y. \& Cahan, P. SingleCellNet: A Computational Tool to Classify Single Cell RNA-Seq Data Across Platforms and Across Species. Cell Syst. 9, 207-213.e2 (2019).

24. Cancer Genome Atlas Network. Comprehensive molecular characterization of human colon and rectal cancer. Nature 487, 330-337 (2012).

25. Zhang, J. et al. International Cancer Genome Consortium Data Portal--a one-stop shop for cancer genomics data. Database (Oxford) 2011, bar026 (2011).

26. Cancer Genome Atlas Network. Comprehensive molecular portraits of human breast tumours. Nature 490, 61-70 (2012).

27. Parker, J. S. et al. Supervised risk predictor of breast cancer based on intrinsic subtypes. J. Clin. Oncol. 27, 1160-1167 (2009). 
1079

1080

1081

1082

1083

1084

1085

1086

1087

1088

1089

1090

1091

1092

1093

1094

1095

1096

28. Wilkerson, M. D. et al. Lung squamous cell carcinoma mRNA expression subtypes are reproducible, clinically important, and correspond to normal cell types. Clin.

Cancer Res. 16, 4864-4875 (2010).

29. Cancer Genome Atlas Research Network. Electronic address: andrew_aguirre@dfci.harvard.edu \& Cancer Genome Atlas Research Network. Integrated genomic characterization of pancreatic ductal adenocarcinoma. Cancer Cell 32, 185-203.e13 (2017).

30. Cancer Genome Atlas Research Network et al. Integrated genomic characterization of endometrial carcinoma. Nature 497, 67-73 (2013).

31. Cancer Genome Atlas Research Network et al. Integrated genomic characterization of oesophageal carcinoma. Nature 541, 169-175 (2017).

32. Cancer Genome Atlas Network. Comprehensive genomic characterization of head and neck squamous cell carcinomas. Nature 517, 576-582 (2015).

33. Cancer Genome Atlas Research Network. Comprehensive molecular characterization of clear cell renal cell carcinoma. Nature 499, 43-49 (2013).

34. Verhaak, R. G. W. et al. Integrated genomic analysis identifies clinically relevant subtypes of glioblastoma characterized by abnormalities in PDGFRA, IDH1, EGFR, and NF1. Cancer Cell 17, 98-110 (2010).

35. Cancer Genome Atlas Research Network. Comprehensive molecular profiling of lung adenocarcinoma. Nature 511, 543-550 (2014).

36. Hu, B. et al. Gastric cancer: Classification, histology and application of molecular pathology. J. Gastrointest. Oncol. 3, 251-261 (2012).

37. Barretina, J. et al. The Cancer Cell Line Encyclopedia enables predictive modelling of anticancer drug sensitivity. Nature 483, 603-607 (2012).

38. Medico, E. et al. The molecular landscape of colorectal cancer cell lines unveils clinically actionable kinase targets. Nat. Commun. 6, 7002 (2015).

39. Park, J.-G. et al. Characteristics of Cell Lines Established from Human Colorectal Carcinoma. Cancer Res. (1987).

40. Jerby-Arnon, L. et al. A cancer cell program promotes T cell exclusion and resistance to checkpoint blockade. Cell 175, 984-997.e24 (2018).

41. Darmanis, S. et al. Single-Cell RNA-Seq Analysis of Infiltrating Neoplastic Cells at the Migrating Front of Human Glioblastoma. Cell Rep. 21, 1399-1410 (2017).

42. Patel, A. P. et al. Single-cell RNA-seq highlights intratumoral heterogeneity in primary glioblastoma. Science 344, 1396-1401 (2014).

43. Xu, B. et al. Regulation of endometrial receptivity by the highly expressed HOXA9, HOXA11 and HOXD10 HOX-class homeobox genes. Hum. Reprod. 29, 781-790 (2014).

44. Raines, A. M. et al. Recombineering-based dissection of flanking and paralogous Hox gene functions in mouse reproductive tracts. Development 140, 2942-2952 (2013).

45. Netinatsunthorn, W., Hanprasertpong, J., Dechsukhum, C., Leetanaporn, R. \& Geater, A. WT1 gene expression as a prognostic marker in advanced serous epithelial ovarian carcinoma: an immunohistochemical study. BMC Cancer 6, 90 (2006).

46. Kelly, Z. et al. The prognostic significance of specific HOX gene expression patterns in ovarian cancer. Int. J. Cancer 139, 1608-1617 (2016). 
1097

1098

1099

1100

1101

1102

1103

1104

1105

1106

1107

1108

1109

1110

1111

1112

1113

1114

1115

1116

1117

1118

1119

1120

1121

1122

1123

1124

1125

1126

1127

1128

1129

1130

1131

1132

1133

1134

1135

1136

1137

1138

1139

1140

1141

1142

47. Cancer Genome Atlas Research Network. Integrated genomic analyses of ovarian carcinoma. Nature 474, 609-615 (2011).

48. Wiegand, K. C. et al. ARID1A mutations in endometriosis-associated ovarian carcinomas. N. Engl. J. Med. 363, 1532-1543 (2010).

49. Murray, M. J. et al. LIN28 Expression in malignant germ cell tumors downregulates let-7 and increases oncogene levels. Cancer Res. 73, 4872-4884 (2013).

50. Biton, A. et al. Independent component analysis uncovers the landscape of the bladder tumor transcriptome and reveals insights into luminal and basal subtypes. Cell Rep. 9, 1235-1245 (2014).

51. Fair, W. R., Israeli, R. S. \& Heston, W. D. Prostate-specific membrane antigen. Prostate 32, 140-148 (1997).

52. Black, J. D., English, D. P., Roque, D. M. \& Santin, A. D. Targeted therapy in uterine serous carcinoma: an aggressive variant of endometrial cancer. Womens Health (Lond. Engl.) 10, 45-57 (2014).

53. Yang, S., Thiel, K. W. \& Leslie, K. K. Progesterone: the ultimate endometrial tumor suppressor. Trends Endocrinol. Metab. 22, 145-152 (2011).

54. Huszar, M. et al. Up-regulation of L1CAM is linked to loss of hormone receptors and E-cadherin in aggressive subtypes of endometrial carcinomas. J. Pathol. 220, 551561 (2010).

55. Kozak, J., Wdowiak, P., Maciejewski, R. \& Torres, A. A guide for endometrial cancer cell lines functional assays using the measurements of electronic impedance. Cytotechnology 70, 339-350 (2018).

56. Korch, C. et al. DNA profiling analysis of endometrial and ovarian cell lines reveals misidentification, redundancy and contamination. Gynecol. Oncol. 127, 241-248 (2012).

57. Wu, D. et al. Gene-expression data integration to squamous cell lung cancer subtypes reveals drug sensitivity. Br. J. Cancer 109, 1599-1608 (2013).

58. Walter, V. et al. Molecular subtypes in head and neck cancer exhibit distinct patterns of chromosomal gain and loss of canonical cancer genes. PLoS One 8, e56823 (2013).

59. Adeegbe, D. O. et al. BET Bromodomain Inhibition Cooperates with PD-1 Blockade to Facilitate Antitumor Response in Kras-Mutant Non-Small Cell Lung Cancer. Cancer Immunol Res 6, 1234-1245 (2018).

60. Blaisdell, A. et al. Neutrophils oppose uterine epithelial carcinogenesis via debridement of hypoxic tumor cells. Cancer Cell 28, 785-799 (2015).

61. Fitamant, J. et al. YAP inhibition restores hepatocyte differentiation in advanced HCC, leading to tumor regression. Cell Rep. 10, 1692-1707 (2015).

62. Jia, D. et al. Crebbp loss drives small cell lung cancer and increases sensitivity to HDAC inhibition. Cancer Discov. 8, 1422-1437 (2018).

63. Kress, T. R. et al. Identification of MYC-Dependent Transcriptional Programs in Oncogene-Addicted Liver Tumors. Cancer Res. 76, 3463-3472 (2016).

64. Li, L. et al. GKAP acts as a genetic modulator of NMDAR signaling to govern invasive tumor growth. Cancer Cell 33, 736-751.e5 (2018).

65. Mollaoglu, G. et al. The Lineage-Defining Transcription Factors SOX2 and NKX2-1 Determine Lung Cancer Cell Fate and Shape the Tumor Immune Microenvironment. Immunity 49, 764-779.e9 (2018). 
66. Pan, Y. et al. Whole tumor RNA-sequencing and deconvolution reveal a clinicallyprognostic PTEN/PI3K-regulated glioma transcriptional signature. Oncotarget 8, 52474-52487 (2017).

67. Lissanu Deribe, Y. et al. Mutations in the SWI/SNF complex induce a targetable dependence on oxidative phosphorylation in lung cancer. Nat. Med. 24, 1047-1057 (2018).

68. Xu, C. et al. Loss of Lkb1 and Pten leads to lung squamous cell carcinoma with elevated PD-L1 expression. Cancer Cell 25, 590-604 (2014).

69. NCl-Frederick, Frederick, MD. National Laboratory for Cancer Research. The NCl Patient-Derived Models Repository (PDMR). (2019). at <https://pdmr.cancer.gov/>

70. Broutier, L. et al. Human primary liver cancer-derived organoid cultures for disease modeling and drug screening. Nat. Med. 23, 1424-1435 (2017).

71. Lee, S. H. et al. Tumor Evolution and Drug Response in Patient-Derived Organoid Models of Bladder Cancer. Cell 173, 515-528.e17 (2018).

72. Ogawa, J., Pao, G. M., Shokhirev, M. N. \& Verma, I. M. Glioblastoma model using human cerebral organoids. Cell Rep. 23, 1220-1229 (2018).

73. Ben-David, U. et al. Patient-derived xenografts undergo mouse-specific tumor evolution. Nat. Genet. 49, 1567-1575 (2017).

74. Stratton, M. R., Campbell, P. J. \& Futreal, P. A. The cancer genome. Nature 458, 719-724 (2009).

75. Balkwill, F. R., Capasso, M. \& Hagemann, T. The tumor microenvironment at a glance. J. Cell Sci. 125, 5591-5596 (2012).

76. Lancaster, M. A. \& Knoblich, J. A. Organogenesis in a dish: modeling development and disease using organoid technologies. Science 345, 1247125 (2014).

77. Bregenzer, M. E. et al. Integrated cancer tissue engineering models for precision medicine. PLoS One 14, e0216564 (2019).

78. Wang, D. H. \& Souza, R. F. Biology of Barrett's esophagus and esophageal adenocarcinoma. Gastrointest Endosc Clin N Am 21, 25-38 (2011).

79. Lee, J. et al. Tumor stem cells derived from glioblastomas cultured in bFGF and EGF more closely mirror the phenotype and genotype of primary tumors than do serum-cultured cell lines. Cancer Cell 9, 391-403 (2006).

80. Wenger, S. L. et al. Comparison of established cell lines at different passages by karyotype and comparative genomic hybridization. Biosci. Rep. 24, 631-639 (2004).

81. Ben-David, U. et al. Genetic and transcriptional evolution alters cancer cell line drug response. Nature 560, 325-330 (2018).

82. Cooke, S. L. et al. Genomic analysis of genetic heterogeneity and evolution in highgrade serous ovarian carcinoma. Oncogene 29, 4905-4913 (2010).

83. Hristova, V. A. \& Chan, D. W. Cancer biomarker discovery and translation: proteomics and beyond. Expert Rev Proteomics 16, 93-103 (2019).

84. Dawson, M. A. \& Kouzarides, T. Cancer epigenetics: from mechanism to therapy. Cell 150, 12-27 (2012).

85. Silva, T. C. et al. TCGA Workflow: Analyze cancer genomics and epigenomics data using Bioconductor packages. [version 2; peer review: 1 approved, 2 approved with reservations]. F1000Res. 5, 1542 (2016).

86. Morgan, M., Obenchain, V., Hester, J. \& Pag`es, H. SummarizedExperiment: SummarizedExperiment container. (2018). 
87. Pavlidis, P. \& Noble, W. S. Analysis of strain and regional variation in gene expression in mouse brain. Genome Biol. 2, RESEARCH0042 (2001).

88. Geman, D., d Avignon, C., Naiman, D. Q. \& Winslow, R. L. Classifying gene expression profiles from pairwise mRNA comparisons. Stat Appl Genet Mol Biol 3, Article19 (2004).

89. Krstajic, D., Buturovic, L. J., Leahy, D. E. \& Thomas, S. Cross-validation pitfalls when selecting and assessing regression and classification models. J. Cheminform. 6, 10 (2014).

90. Lipton, Z. C., Elkan, C. \& Naryanaswamy, B. Optimal Thresholding of Classifiers to Maximize F1 Measure. Mach. Learn. Knowl. Discov. Databases 8725, 225-239 (2014).

91. Grow, E. J. et al. Intrinsic retroviral reactivation in human preimplantation embryos and pluripotent cells. Nature 522, 221-225 (2015).

92. Kolde, R. pheatmap: Pretty Heatmaps. (CRAN, 2019).

93. Wickham, H. ggplot2 - Elegant Graphics for Data Analysis . (Springer-Verlag New York, 2016). doi:10.1007/978-0-387-98141-3

94. Gu, Z., Eils, R. \& Schlesner, M. Complex heatmaps reveal patterns and correlations in multidimensional genomic data. Bioinformatics 32, 2847-2849 (2016).

95. Yoshihara, K. et al. Inferring tumour purity and stromal and immune cell admixture from expression data. Nat. Commun. 4, 2612 (2013).

96. Kovalchik, S. RISmed: Download Content from NCBI Databases. (CRAN.R-project, 2017). 
bioRxiv preprint doi: https://doi.org/10.1101/2020.03.27.012757; this version posted January 9, 2021. The copyright holder for this preprint (which was not certified by peer review) is the author/funder, who has granted bioRxiv a license to display the preprint in perpetuity. It is made Figure 1 available under aCC-BY-NC-ND 4.0 International license.

A

RNA-seq from ...
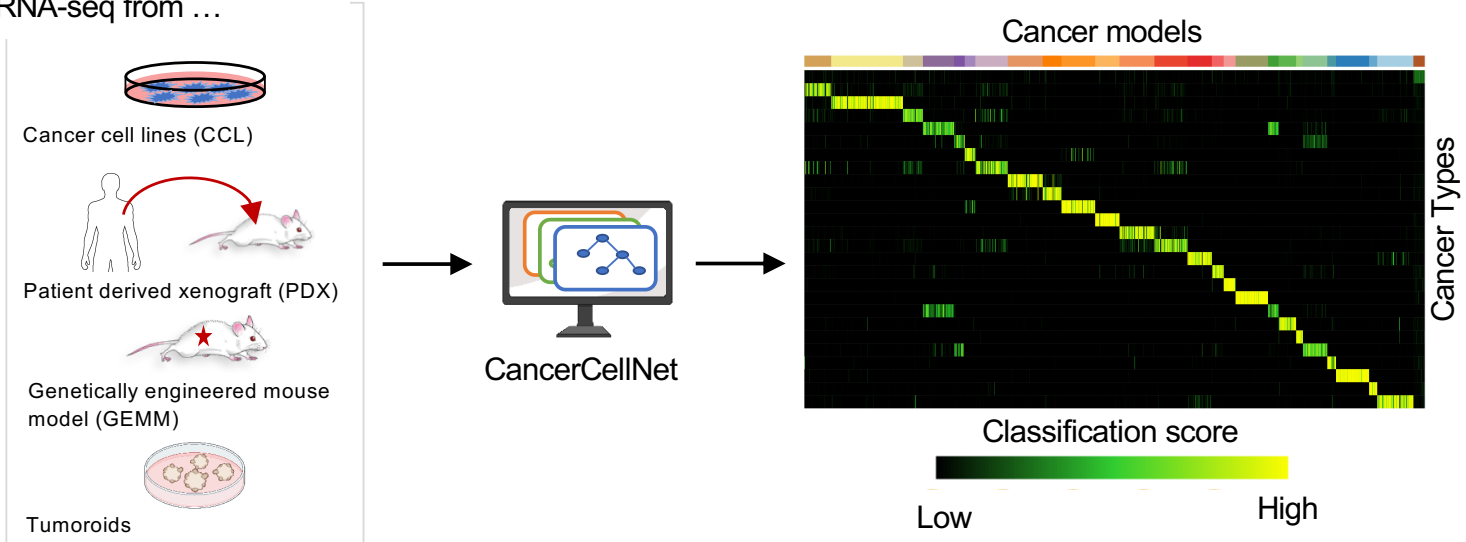

B

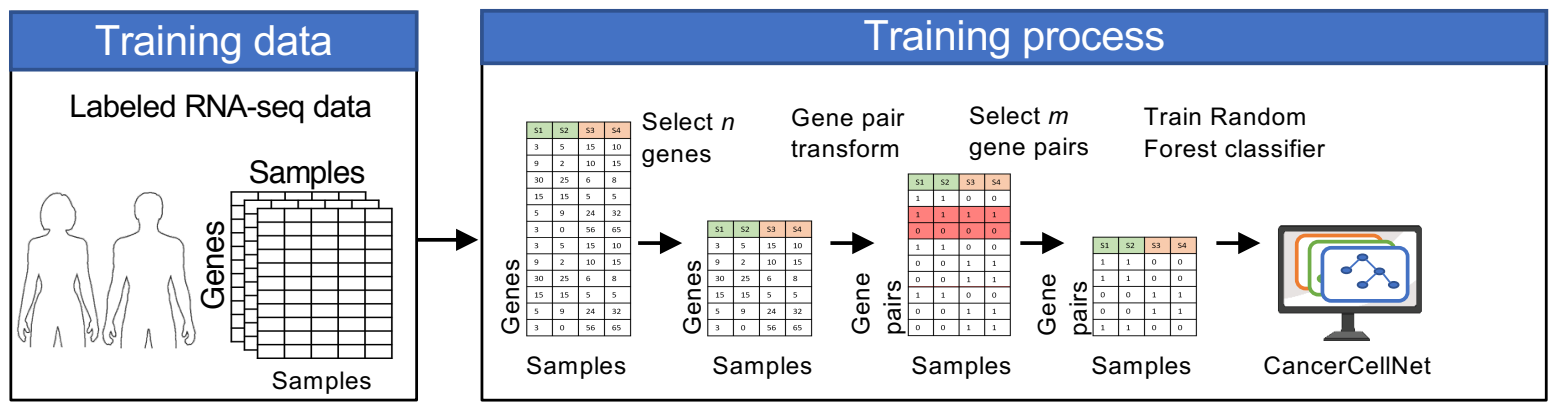

C

(1) Set parameters $n, m$

(2) Randomly select $2 / 3$ TCGA data; run training process

(3) Assess performance on $1 / 3$ held out data

(4) Repeat steps (2-3) 5 times

(5) Repeat steps (1-4) for each parameter set

E

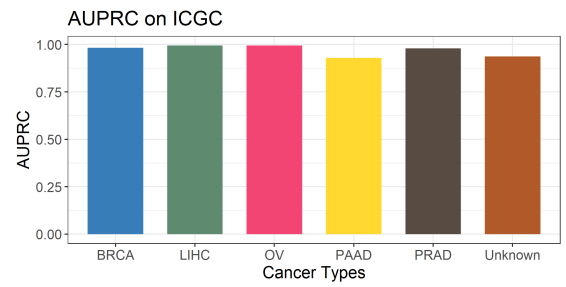

L

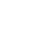

Select parameter set with maximum mean AUPRC. Train on all TCGA data

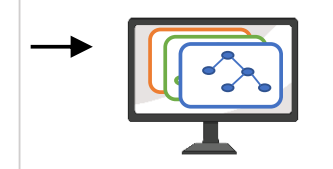

CancerCellNet

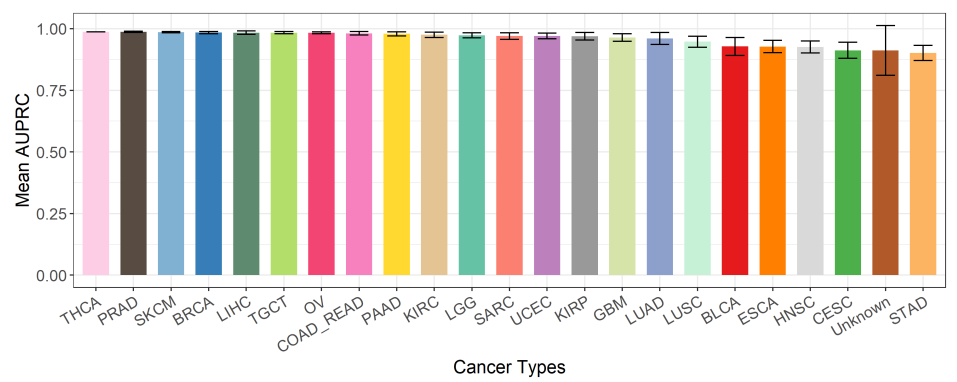

Cancer Types

D 
bioRxiv preprint doi: https://doi.org/10.1101/2020.03.27.012757; this version posted January 9, 2021. The copyright holder for this preprint (which was not certified by peer review) is the author/funder, who has granted bioRxiv a license to display the preprint in perpetuity. It is made Figure 2 available under aCC-BY-NC-ND 4.0 International license.

A

Cancer Cell Lines Classification

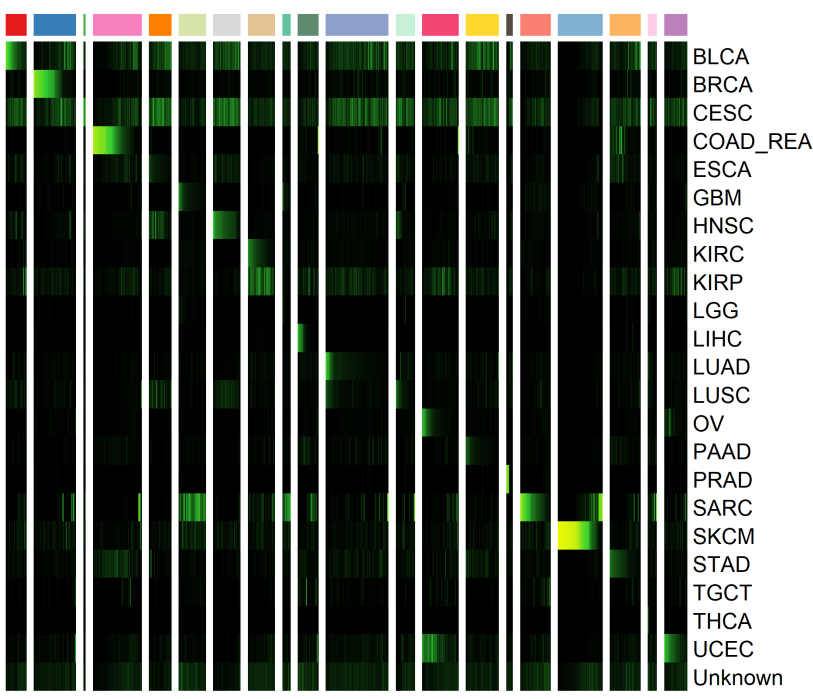

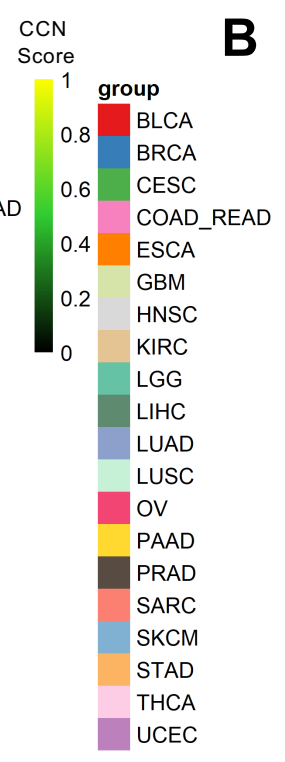

B

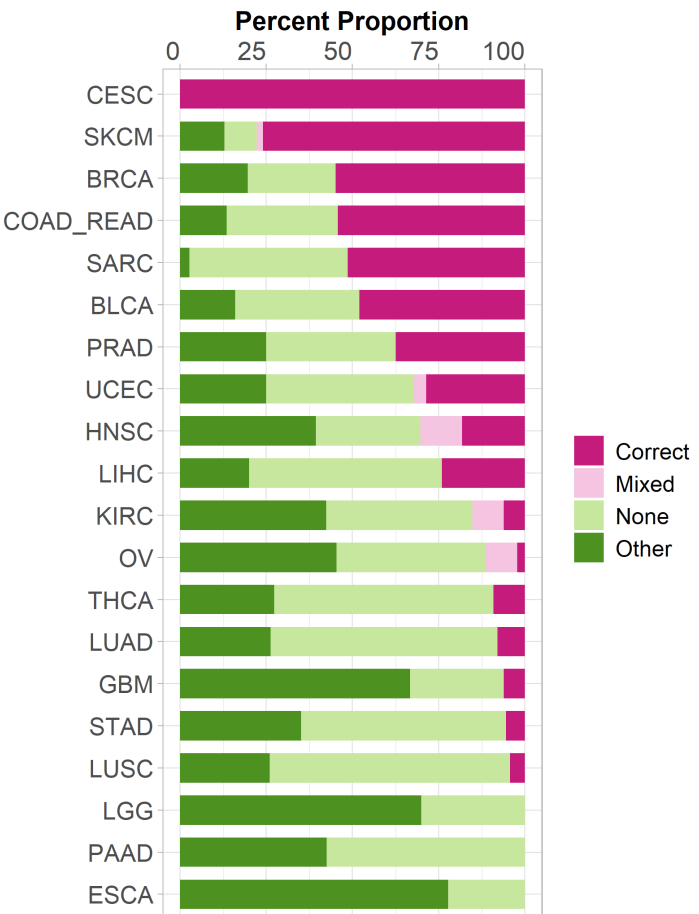

C

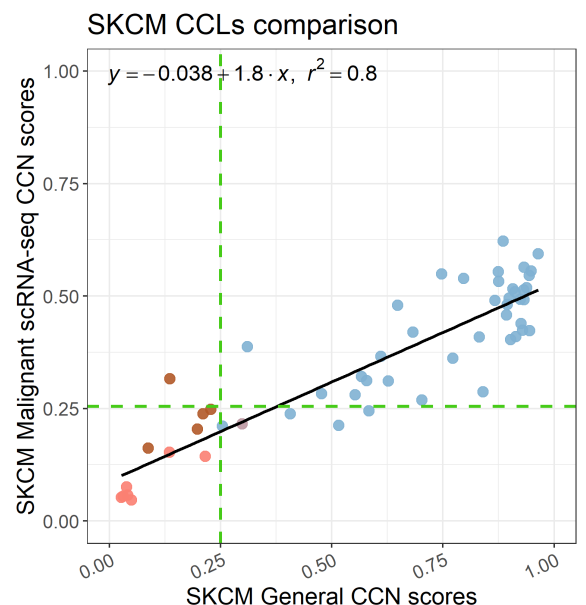

E GBM CCLs comparison

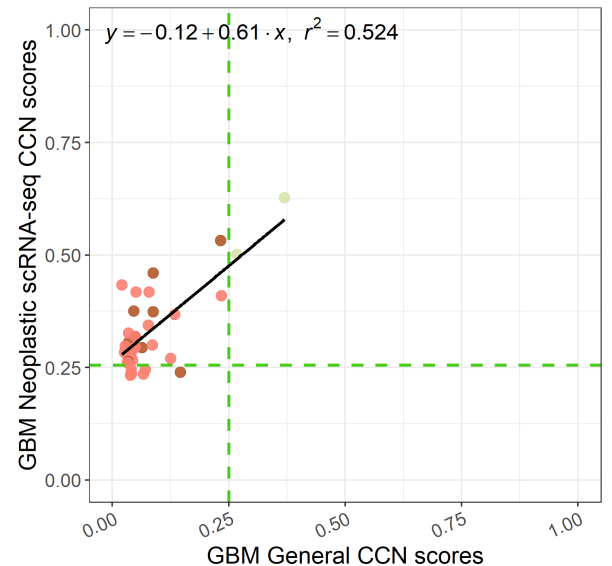

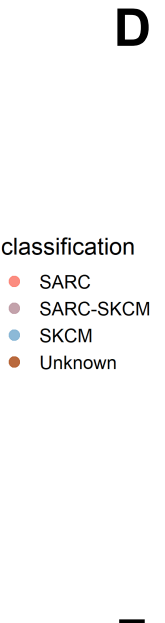

F
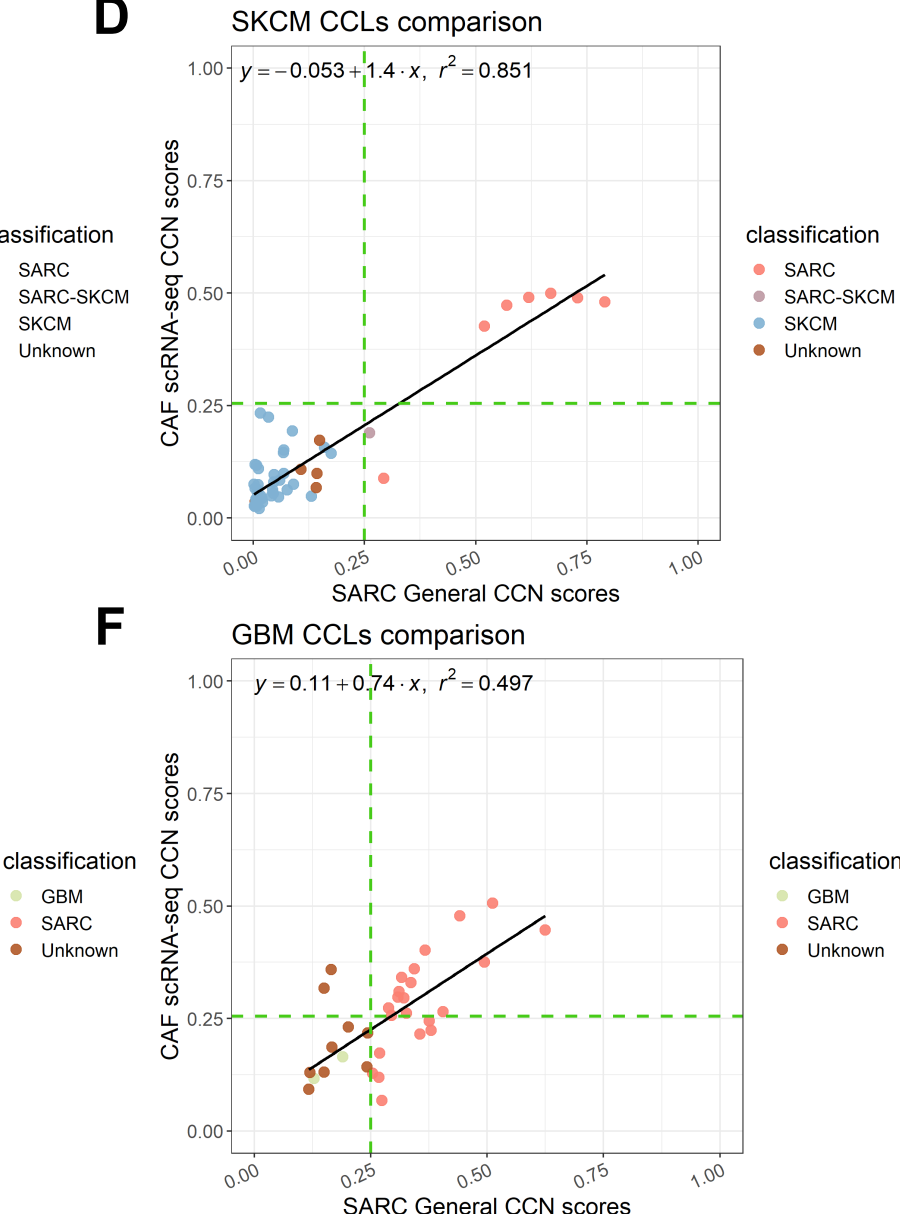

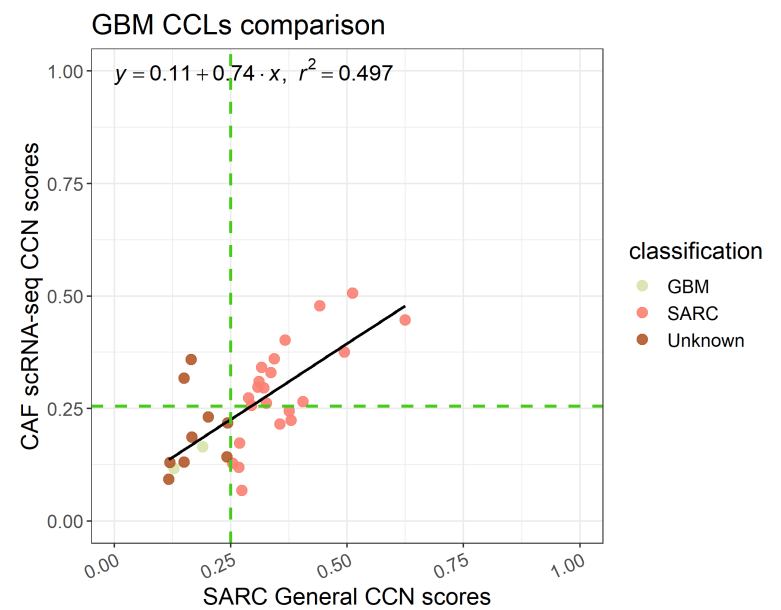




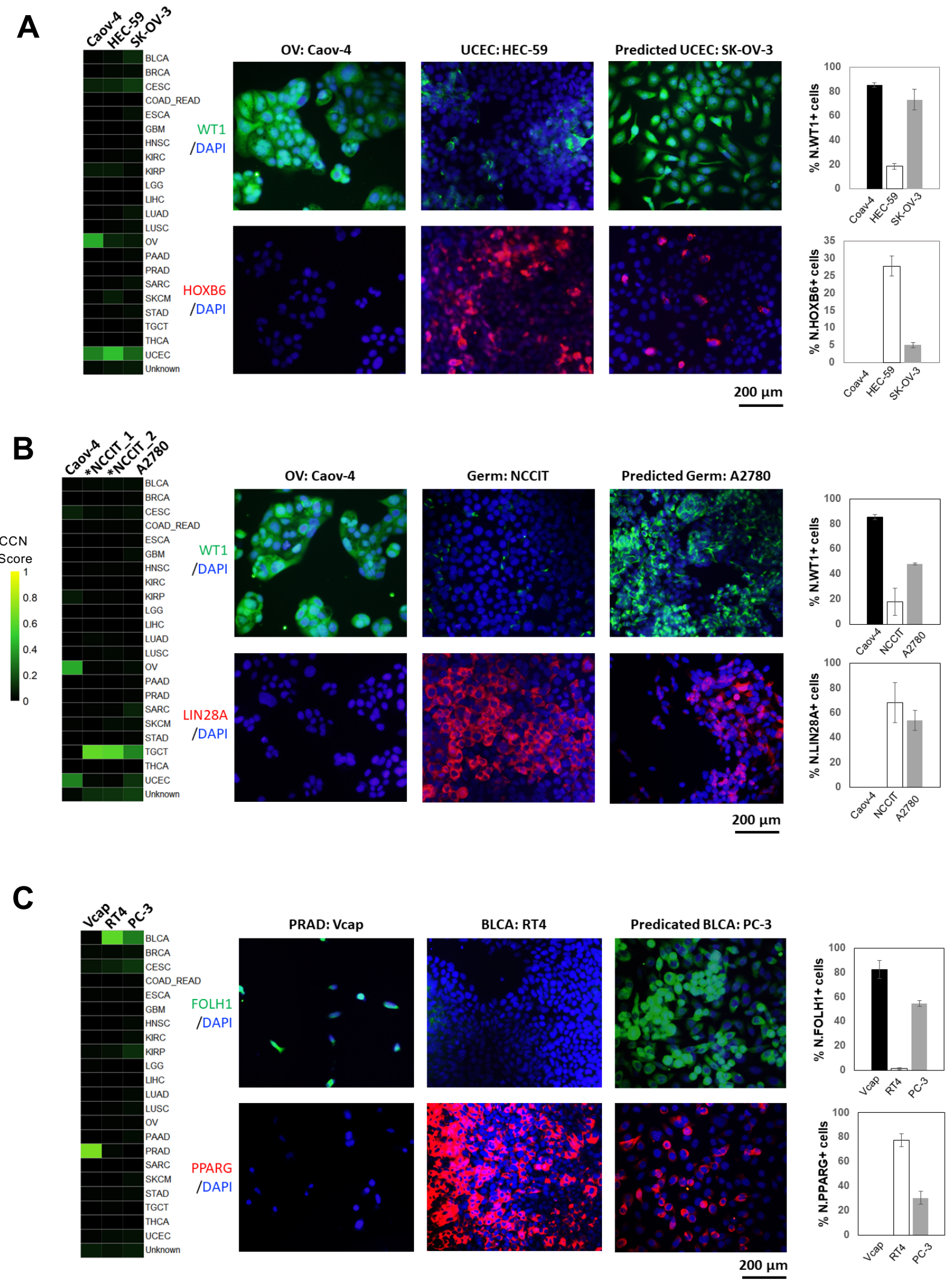


bioRxiv preprint doi: https://doi.org/10.1101/2020.03.27.012757; this version posted January 9, 2021. The copyright holder for this preprint (which was not certified by peer review) is the author/funder, who has granted bioRxiv a license to display the preprint in perpetuity. It is made Figure 4 available under aCC-BY-NC-ND 4.0 International license.

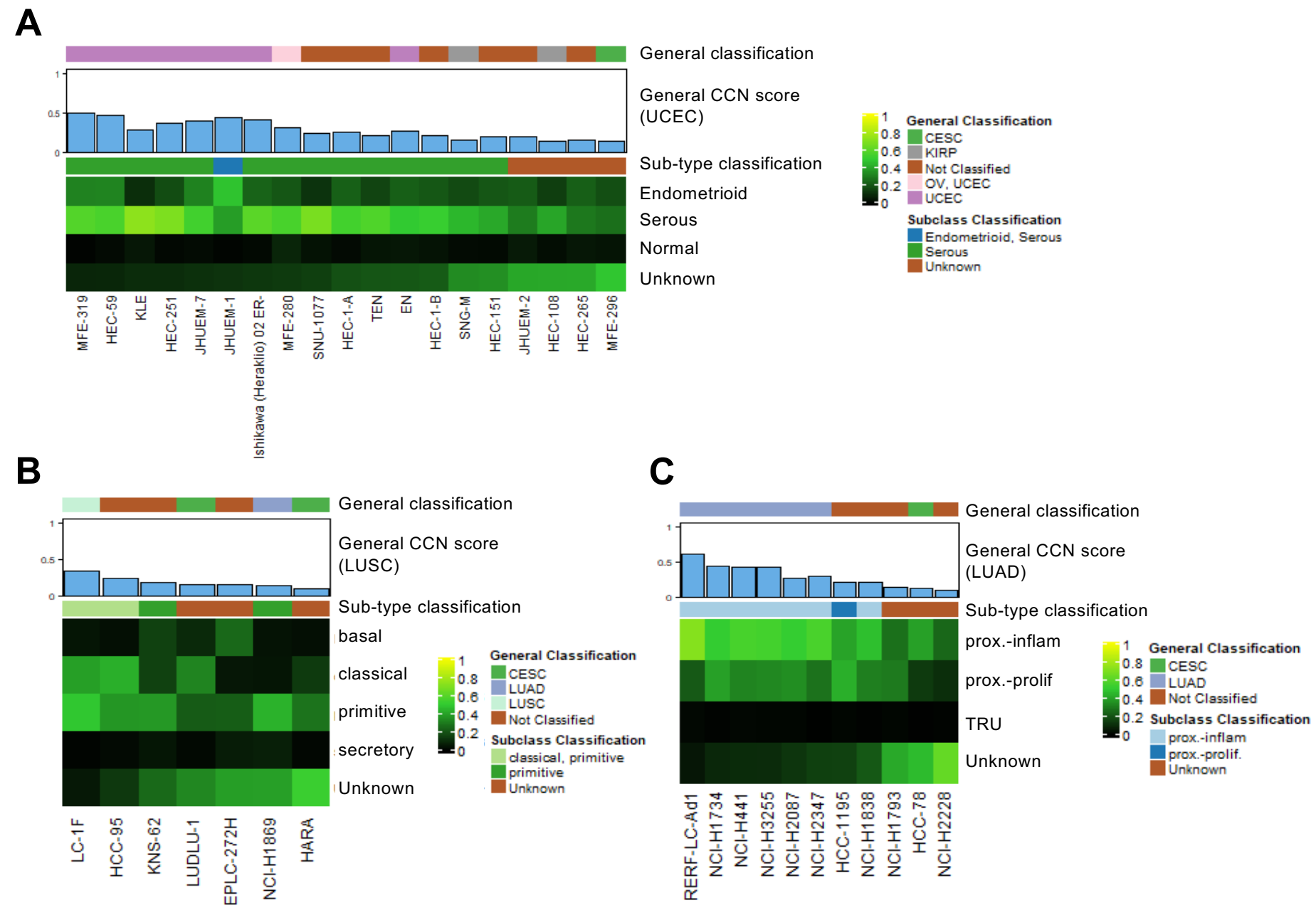

D

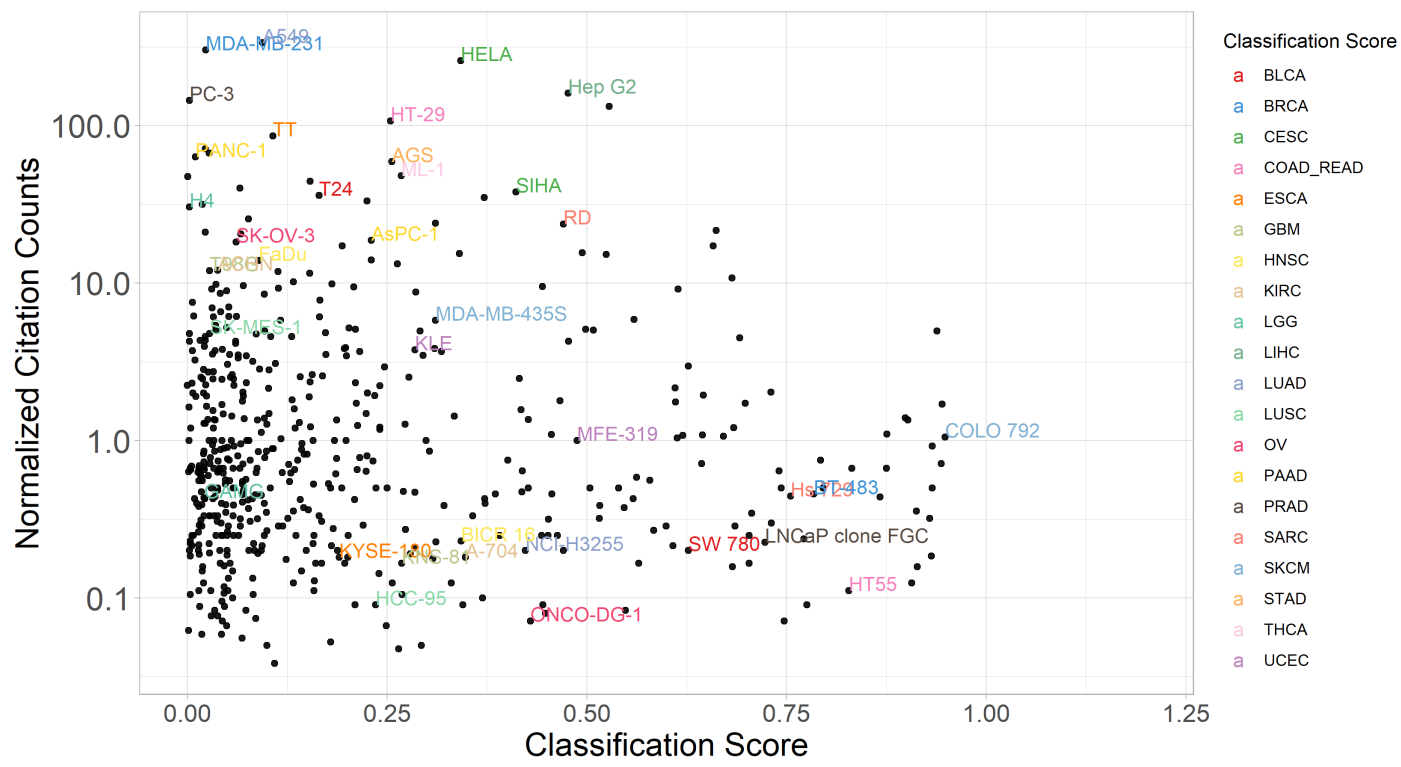


bioRxiv preprint doi: https://doi.org/10.1101/2020.03.27.012757; this version posted January 9, 2021. The copyright holder for this preprint (which was not certified by peer review) is the author/funder, who has granted bioRxiv a license to display the preprint in perpetuity. It is made Figure 5 available under aCC-BY-NC-ND 4.0 International license.
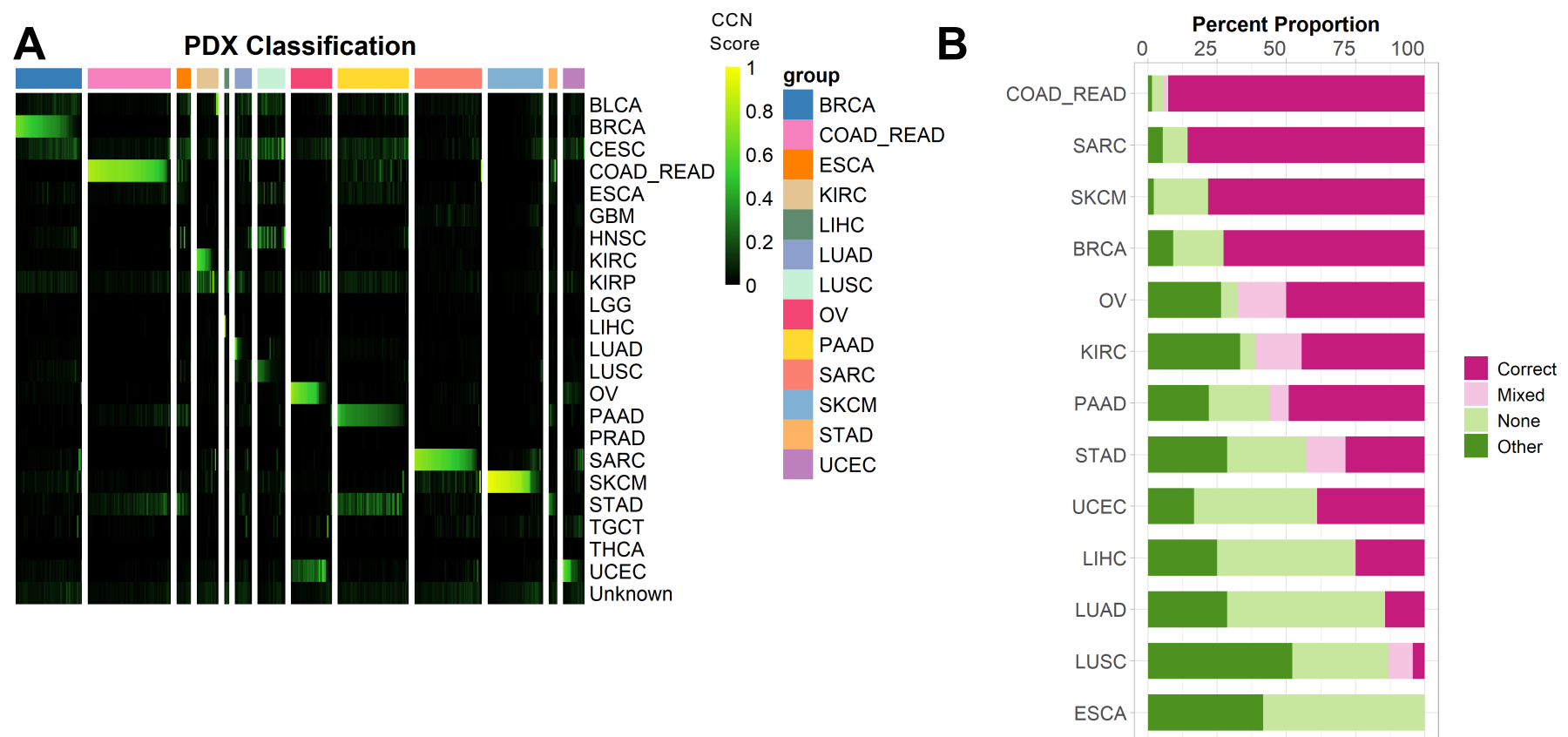

C

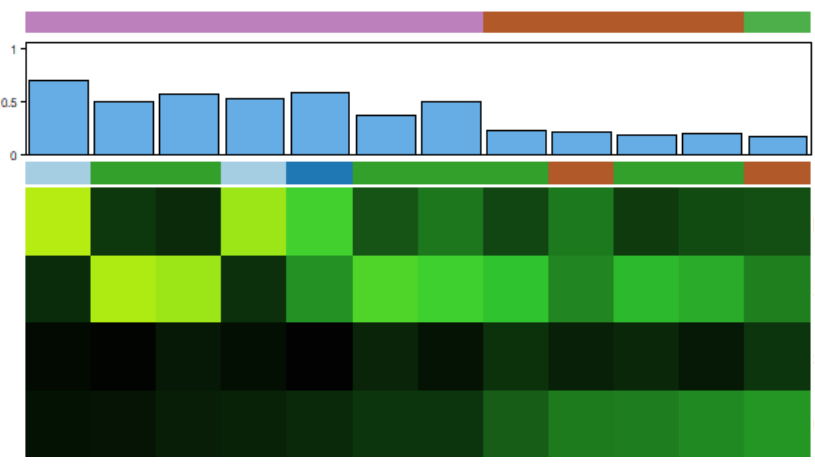

General classification

General CCN score (UCEC)

Sub-type classification

Endometrioid

Serous

Normal

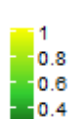

General Classification

CESC

8 Not Classified

Unknown

0.4 Subclass Classification

0.2 Endometrioid

Endometrioid, Serous

Serous

Unknown Unknown

D

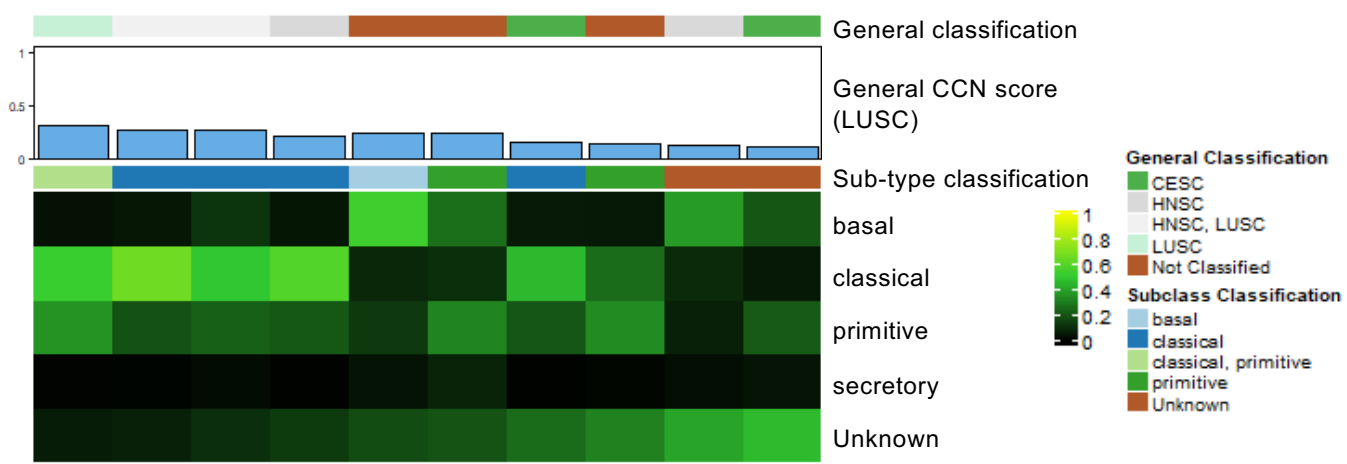

E

General classification

General CCN score

(LUAD)

Sub-type classification

prox.-inflam

prox.-prolif

1 General Classificatio

0.8 LUAD

0.6 Not Classified

-0.4 Subclass Classification

TRU

$0.2 \square$ prox.-inflam

Unknown 

- (which was not certified by peer review) is the author/funder, who has granted bioRxiv a license to display the preprint in perpetuity. It is made Figure 6 available under aCC-BY-NC-ND 4.0 International license.

A

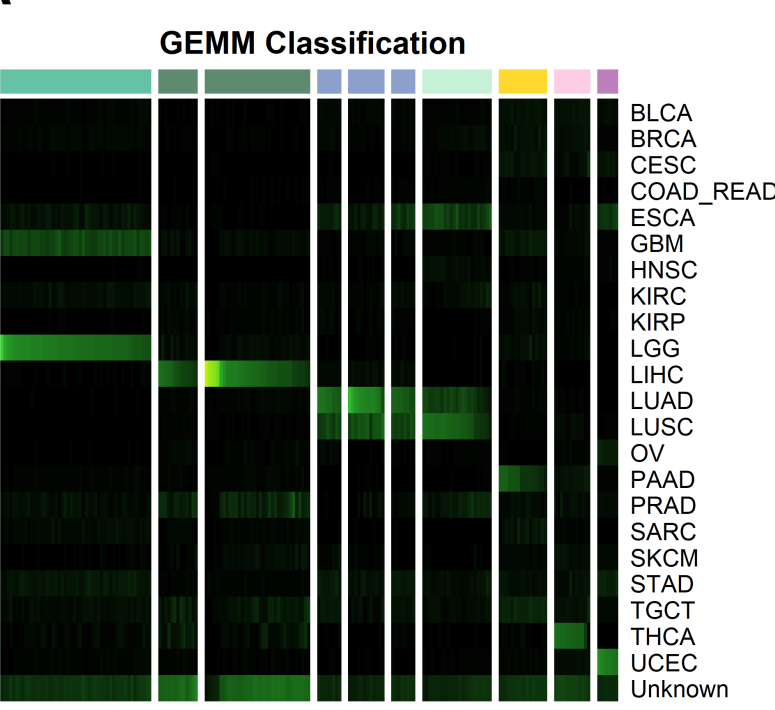

B

$\mathbf{B}$



C

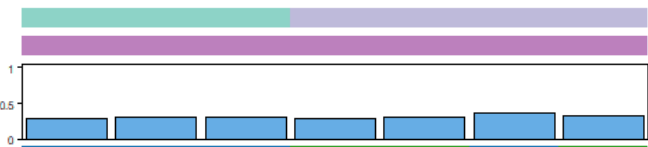

Genotype

General classification

General CCN score

(UCEC)

Sub-type classification

Endometrioid

Genotype

$\operatorname{Pgr}(\alpha e /+\operatorname{Pten}(\operatorname{lox} / \operatorname{lox})$

$0.8 \mathrm{Pgr}(\alpha \mathrm{dr} /+)$ Pten(lox/lox $) \mathrm{Csf} 3 \mathrm{r}(-/-)$

Serous

Normal

Unknown

0.6 General Classification

-0.4 UCEC

-0.2 Subclass Classification

Endometrioid, Serous
Serous

D

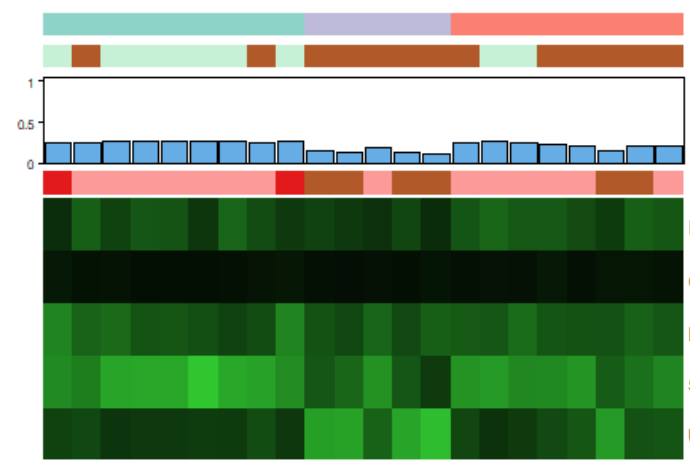

Genotype

General classification

General CCN score

(LUSC)

Sub-type classification Genotype

basal

classical

. Rosa26LSL-Sox2-IRES-GFP;Lkb1fl/fl (SL)

primitive

0.8 General Classification

primitive

secretory

0.4 Not Classified

0.2 Subclass Classification

primitive, secretory

Secretory

Unknown

E

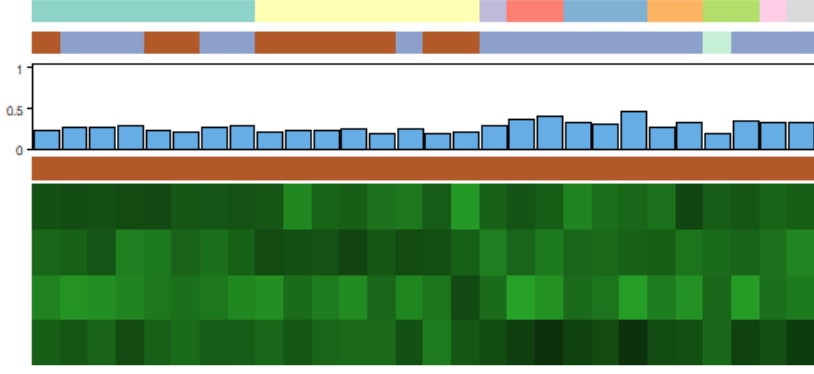

Genotype

General classification

General CCN score

(LUAD)

Sub-type classification prox.-inflam prox.-prolif

TRU

Unknown

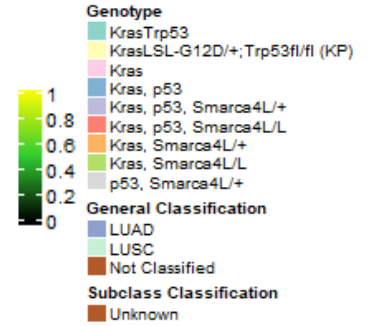

Kras, Smarca4L/+

p53, Smarca4L

General Classification

Subclass Classification

Unknown 
bioRxiv preprint doi: https://doi.org/10.1101/2020.03.27.012757; this version posted January 9, 2021. The copyright holder for this preprint (which was not certified by peer review) is the author/funder, who has granted bioRxiv a license to display the preprint in perpetuity. It is made Figure 7 available under aCC-BY-NC-ND 4.0 International license.

A

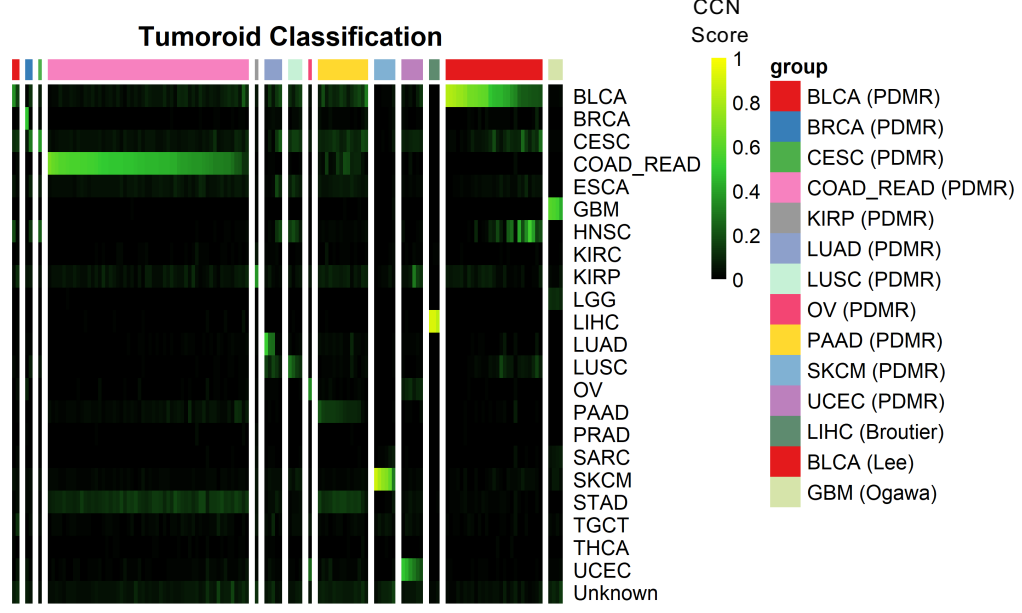

B

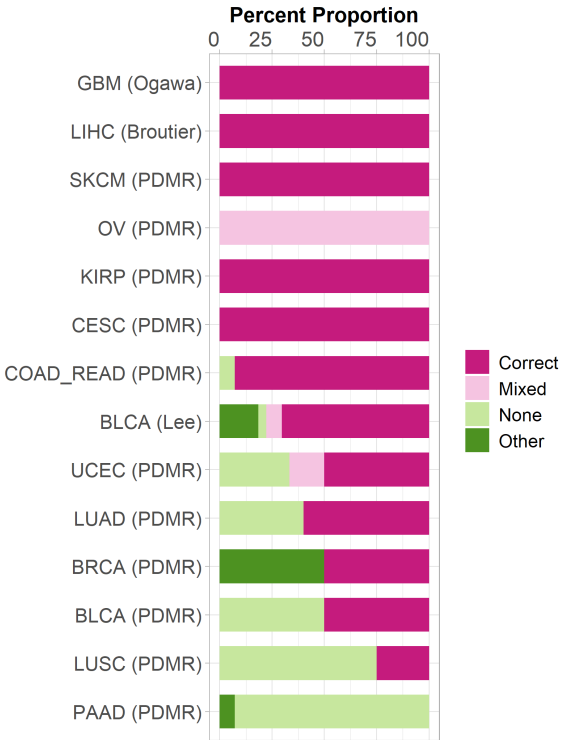

C
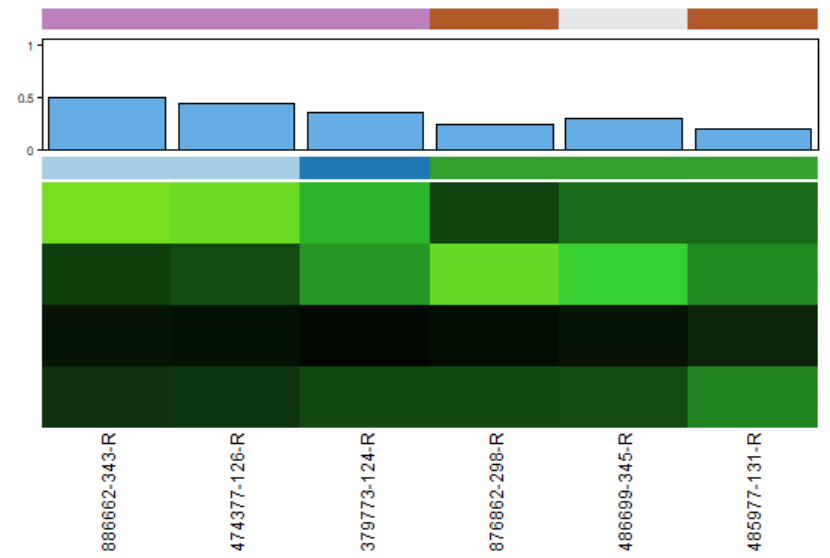

D

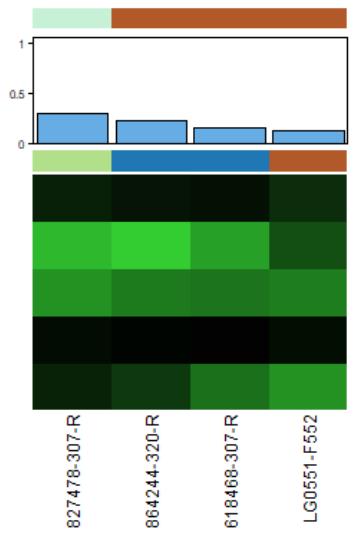

General classification

General CCN score (LUSC)

Sub-type classification

basal

classical

primitive

secretory

Unknown
General classification

General CCN score

(UCEC)

Sub-type classification

Endometrioid General Classification

Serous

KIRP, UCEC

Not Classified

0.4 Subclass Classification

0.4 Endometrioid

0.2 Endometrioid, Serous

Unknown
E

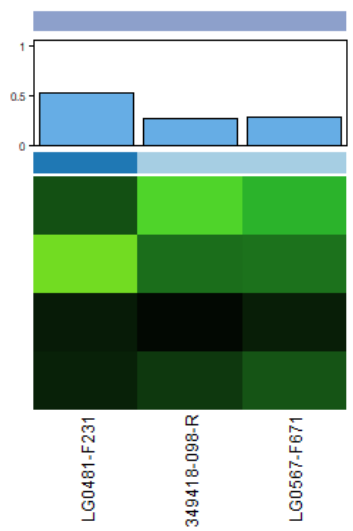

General classification

General CCN score (LUAD)

Sub-type classification LUSC

$0.8 \square$ Not Classified

0.6 Subclass Classification

-0.2 classical

.2 dassical, primitive prox.-inflam

prox.-prolif

TRU

Unknown
General Classification -0.8 LUAD

Subclass Classification

$-0.2 \square$ prox.-inflam 


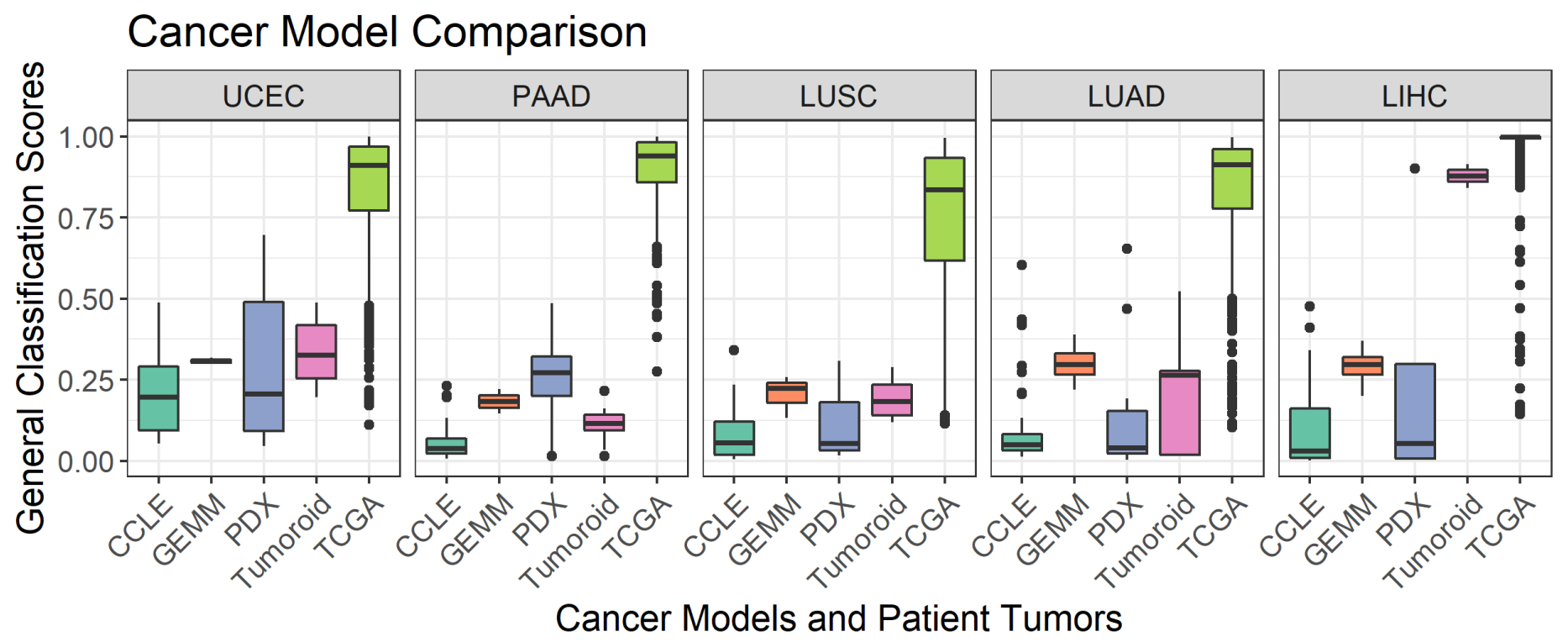


bioRxiv preprint doi: https://doi.org/10.1101/2020.03.27.012757; this version posted January 9, 2021. The copyright holder for this preprint (which was not certified by peer review) is the author/funder, who has granted bioRxiv a license to display the preprint in perpetuity. It is made

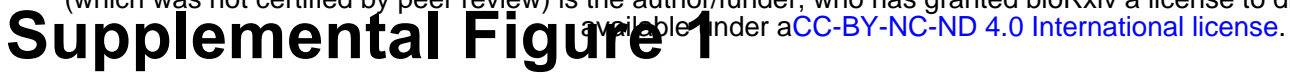

A

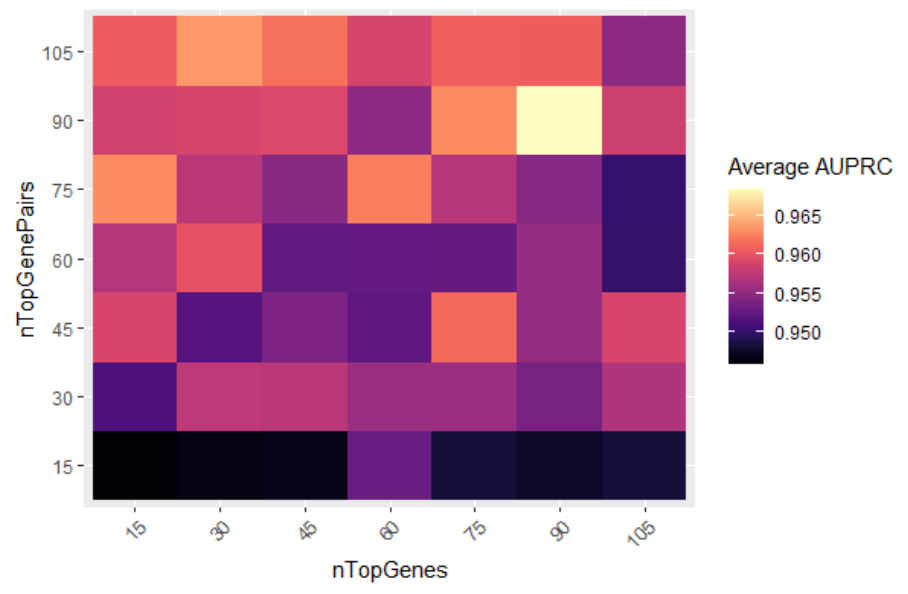

C

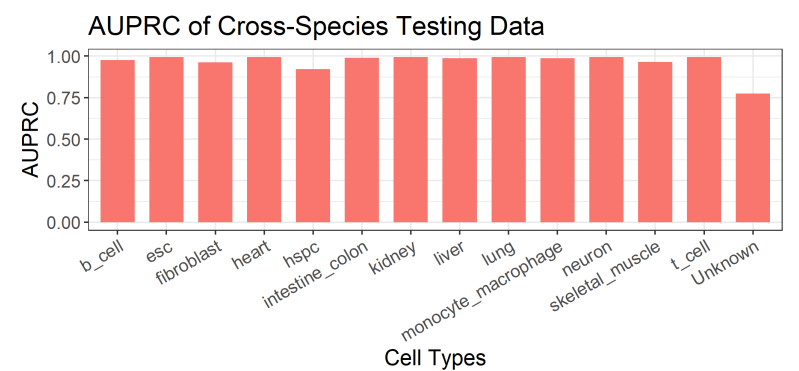

D

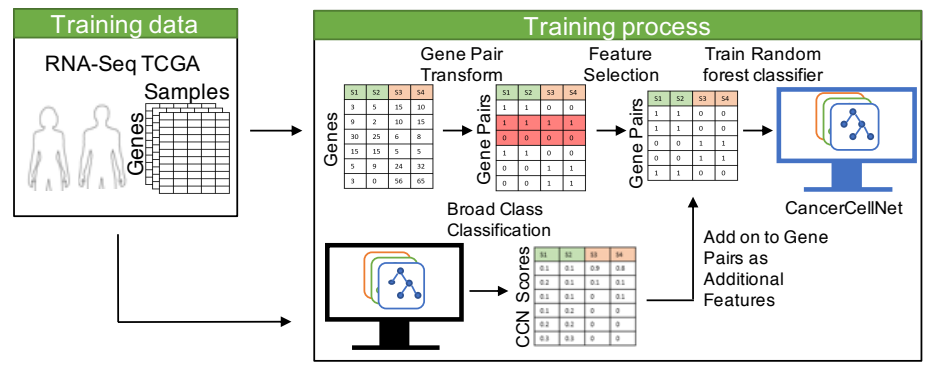

B

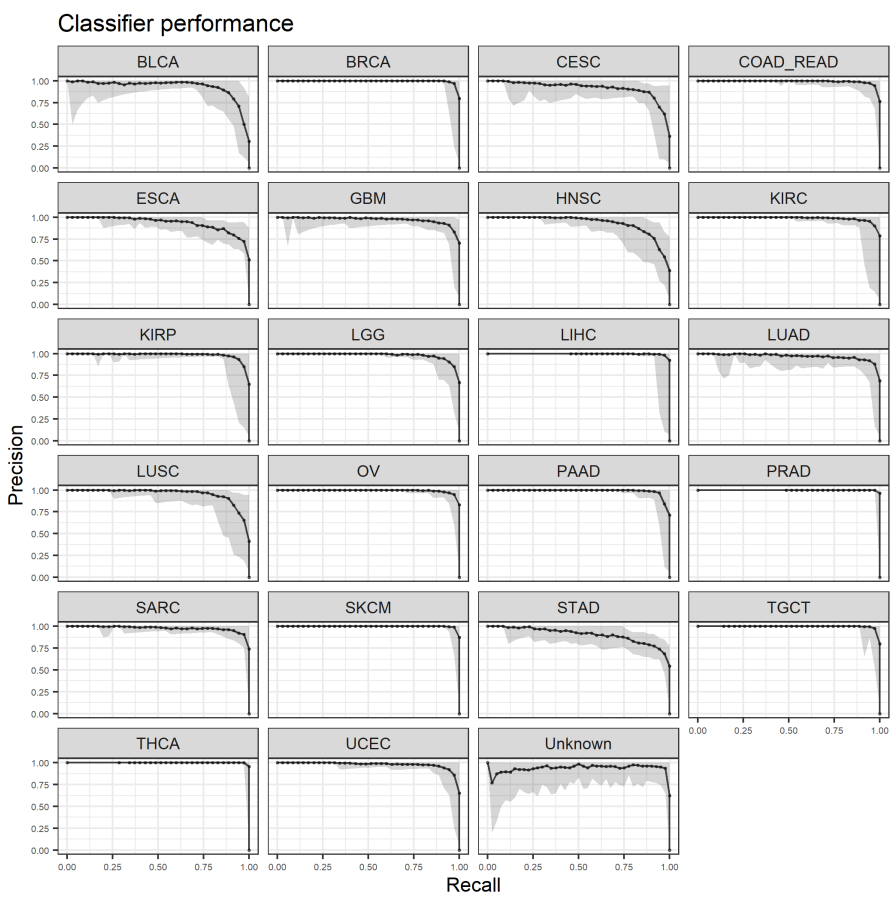

E

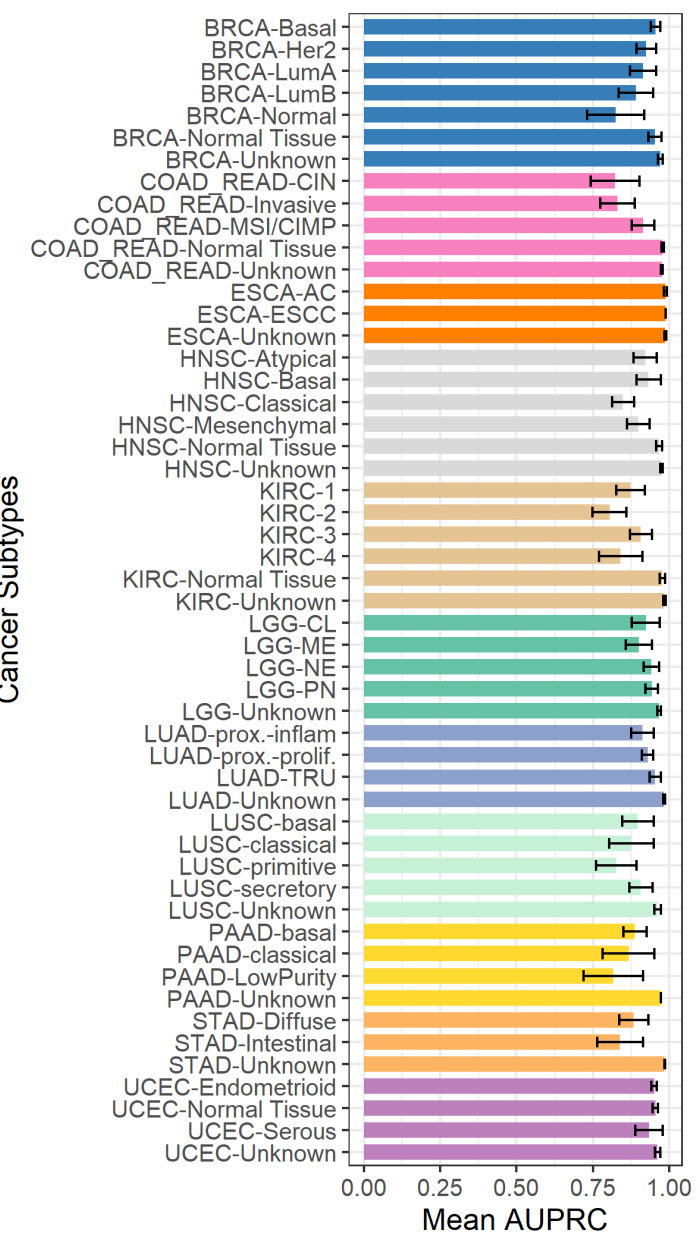


A



C

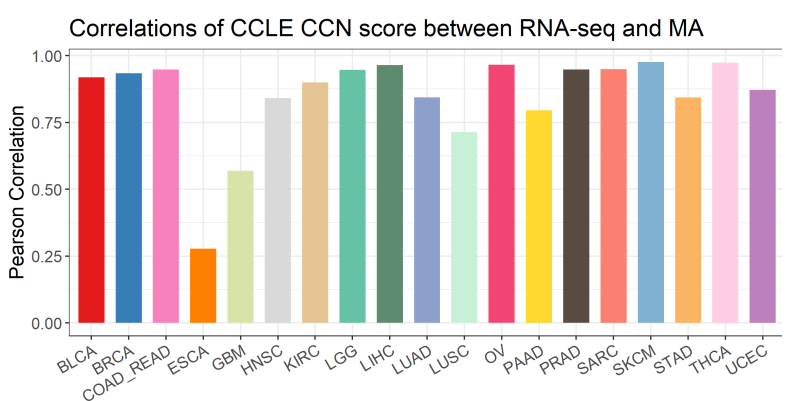

E

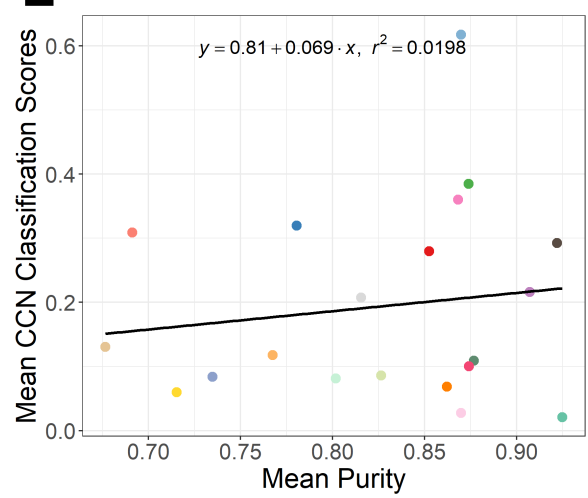

B

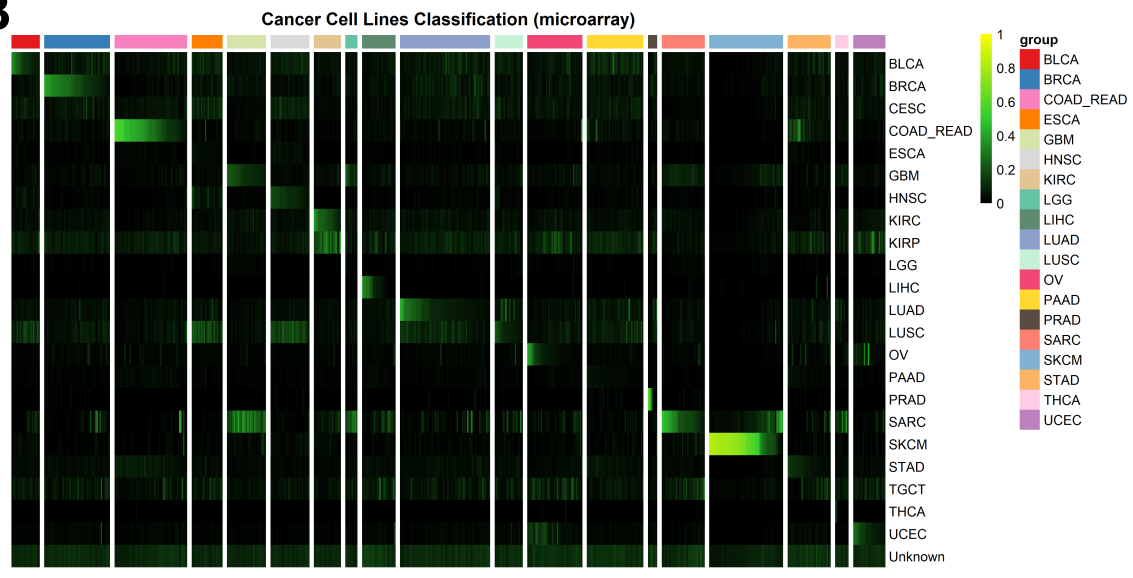

D

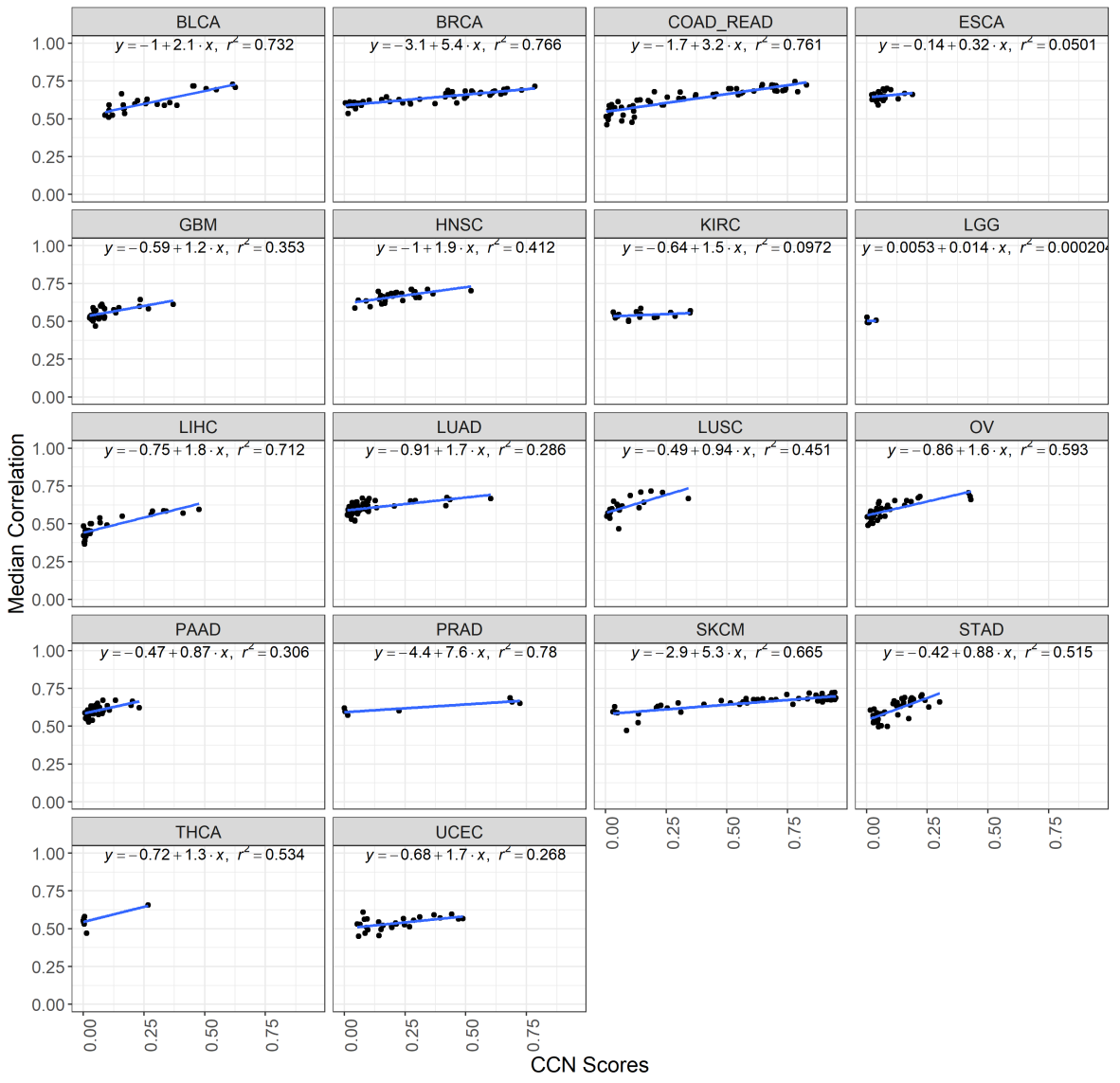


A



B

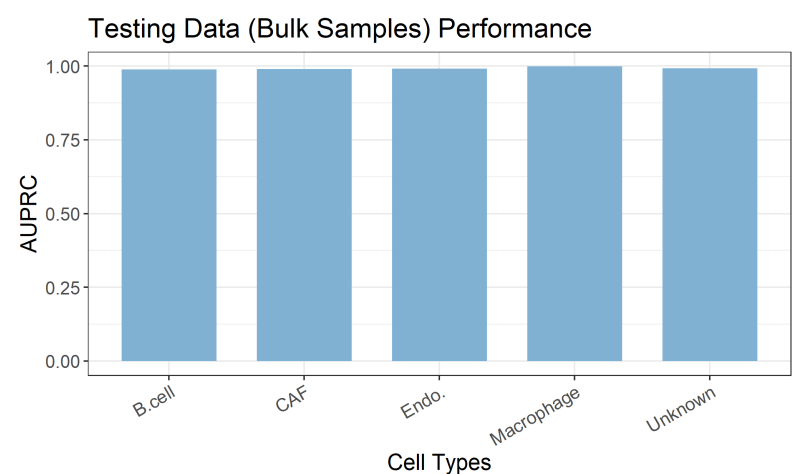

C

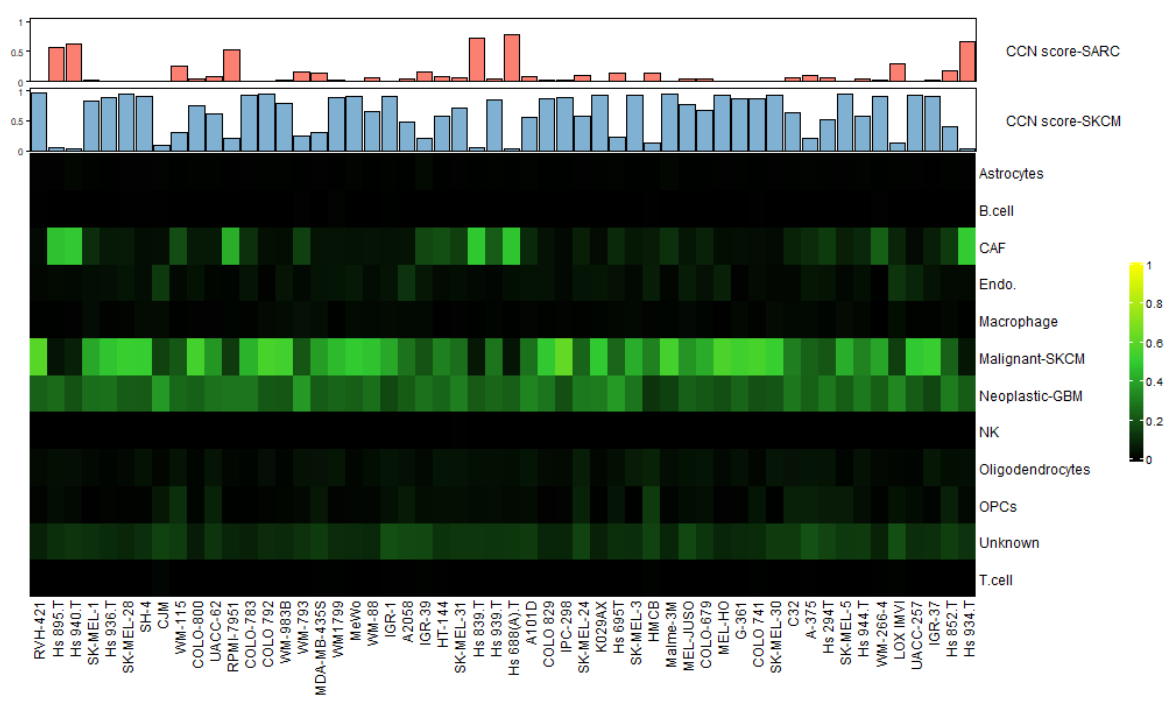

D

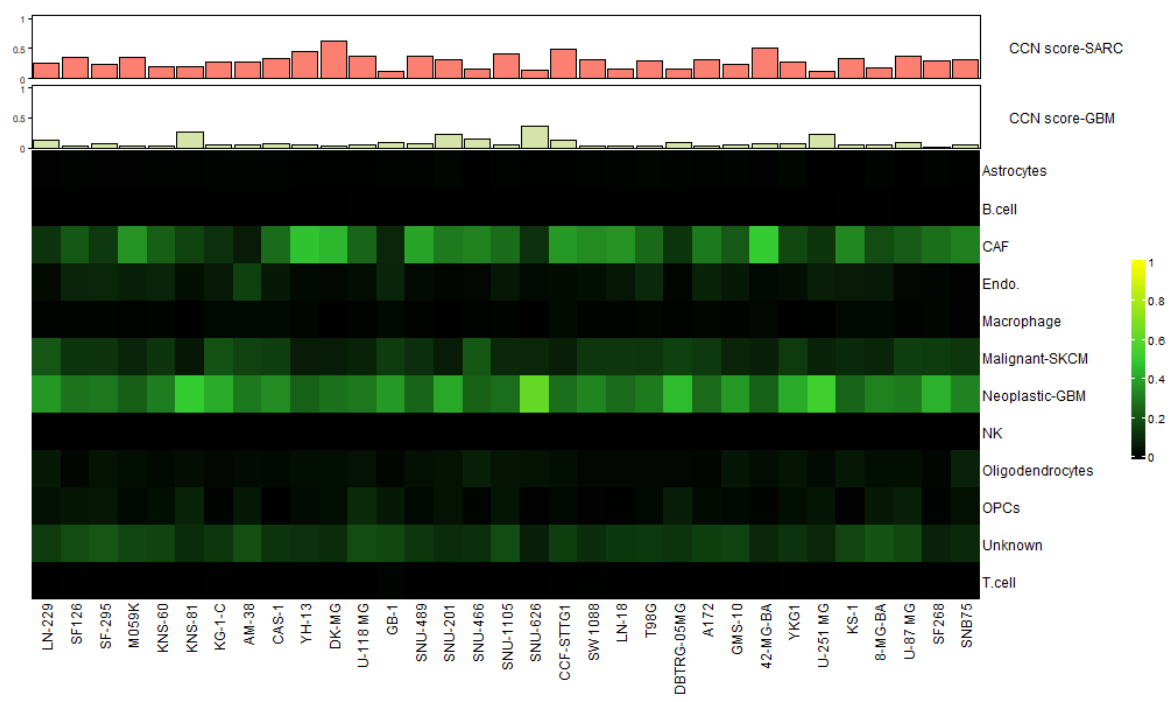



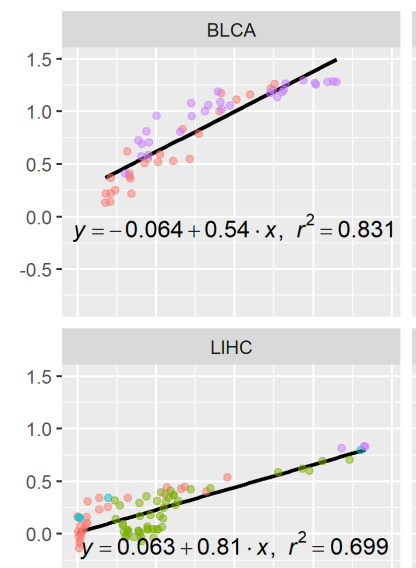

$$
-0.5-
$$
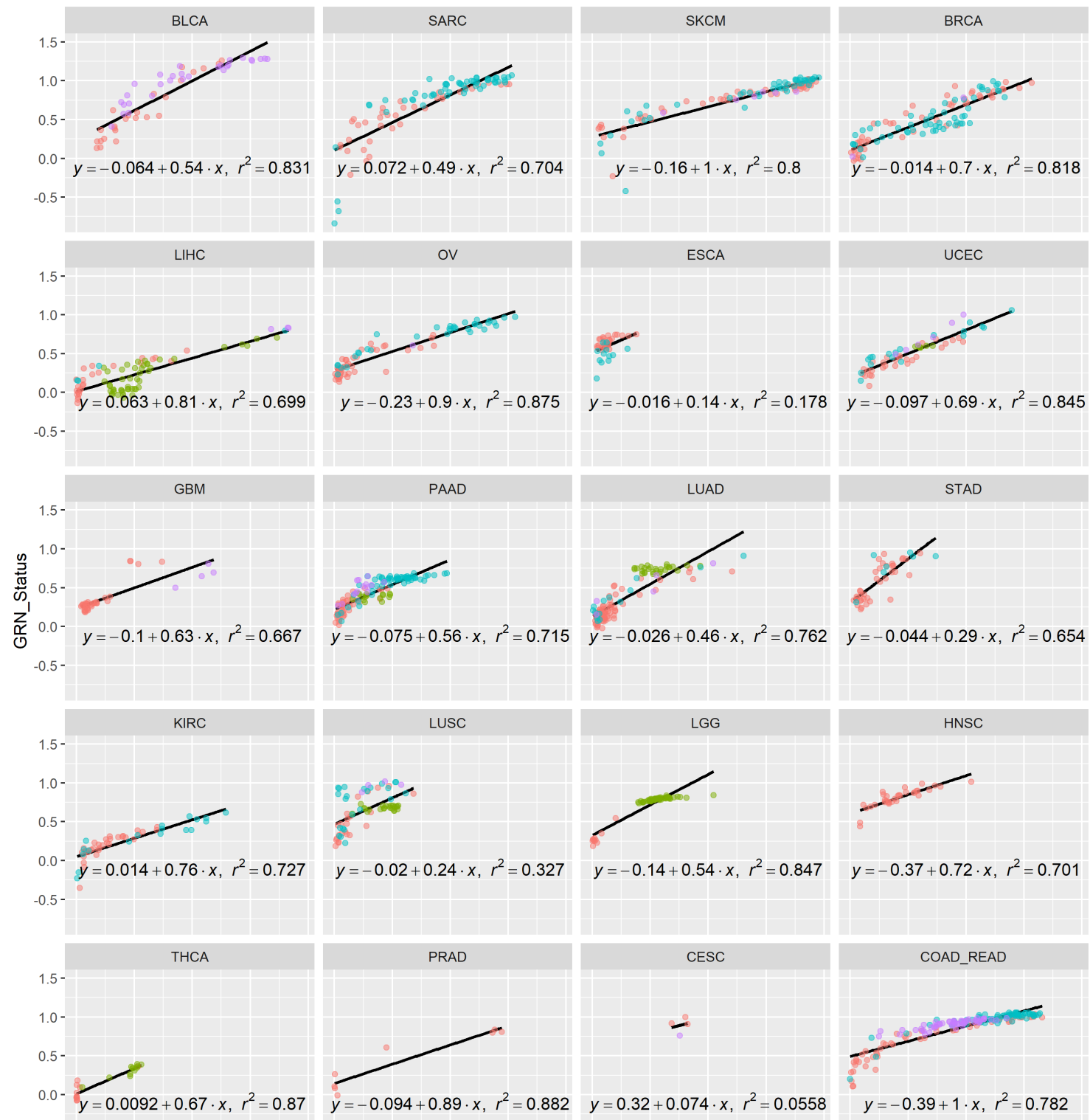

ModelType

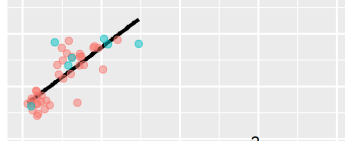

$y=-0.044+0.29 \cdot x, r^{2}=0.654$

GEMM

- PDX $0.5-$

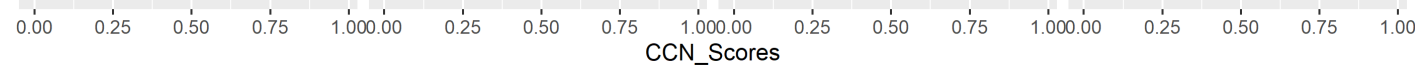




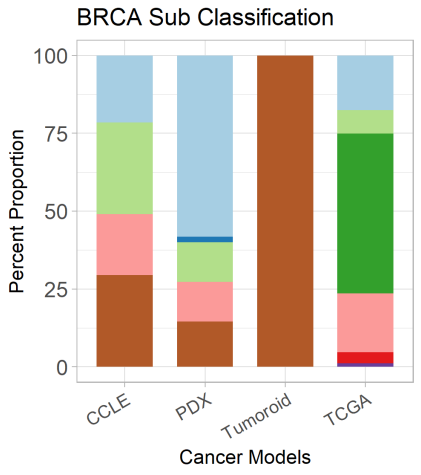

HNSC Sub Classification
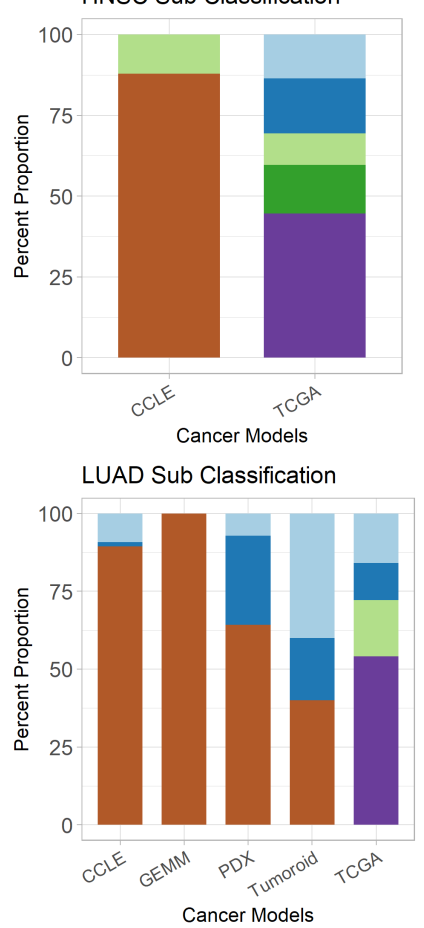

STAD Sub Classification

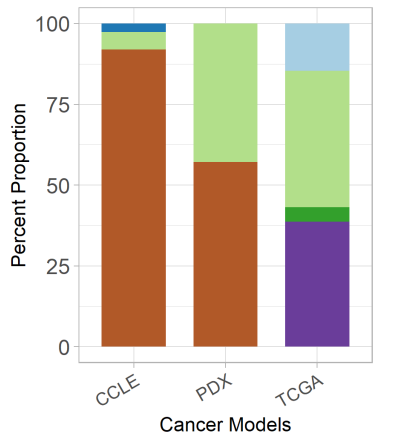

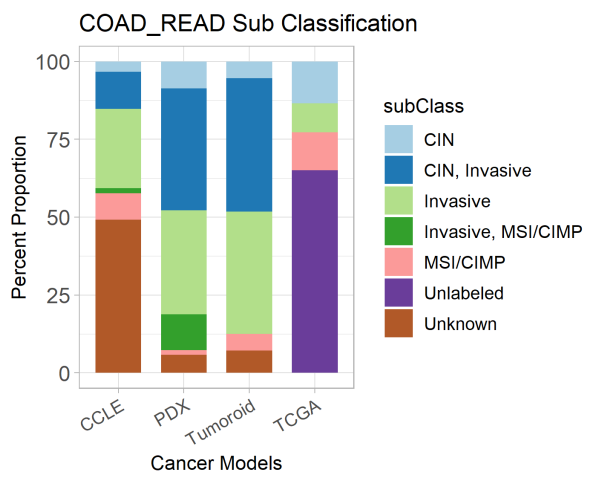
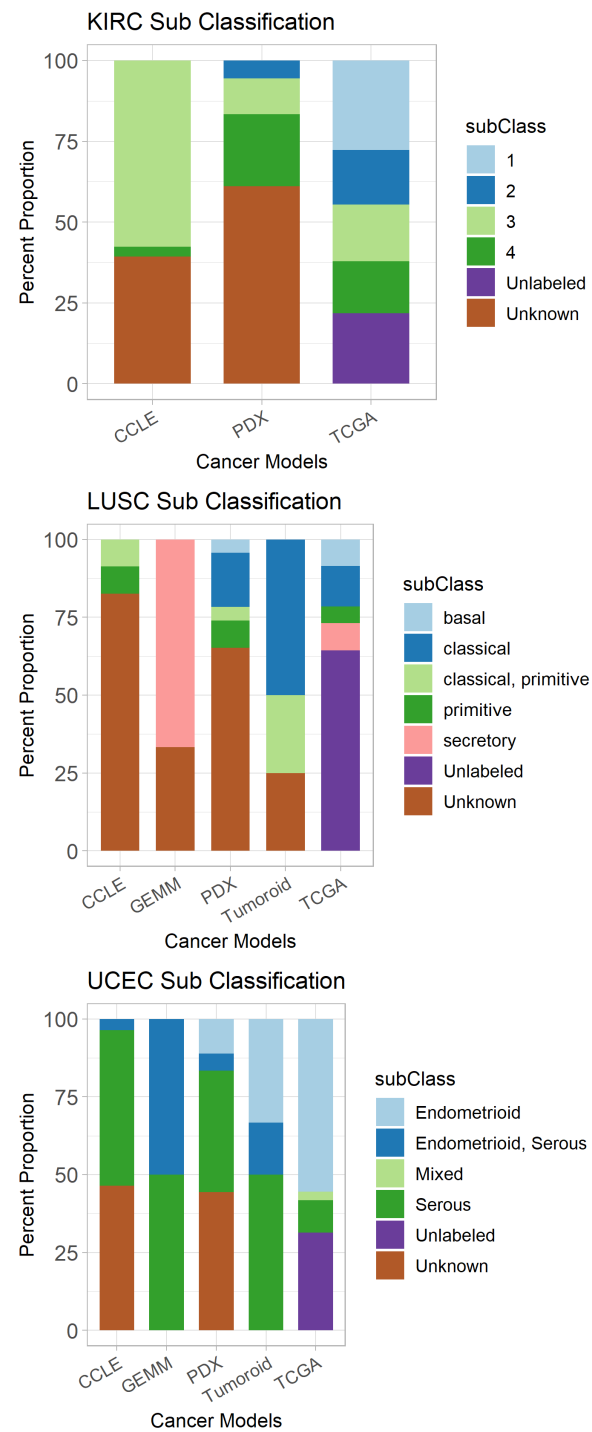
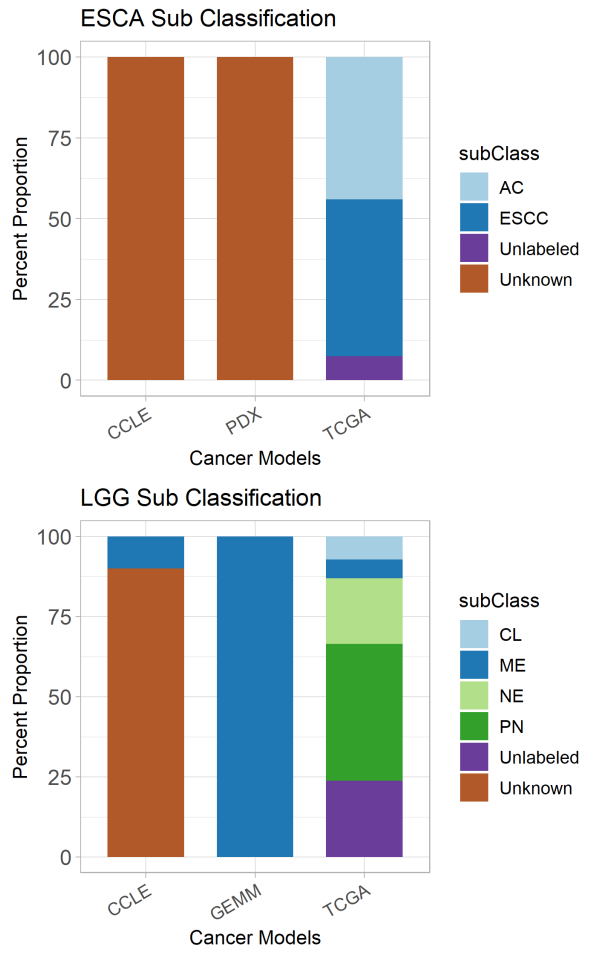

PAAD Sub Classification

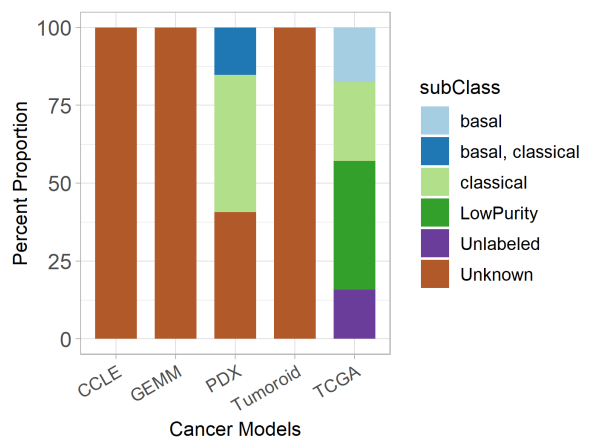

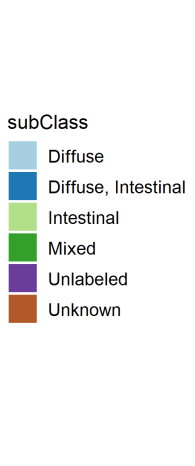

